J. DIFFERENTIAL GEOMETRY

76 (2007) 351-398

\title{
PRINCIPAL BUNDLES ON PROJECTIVE VARIETIES AND THE DONALDSON-UHLENBECK COMPACTIFICATION
}

\author{
V. BALAJI \\ To Peter Newstead on his 65th birthday
}

\begin{abstract}
Let $H$ be a semisimple algebraic group. We prove the semistable reduction theorem for $\mu$-semistable principal $H$-bundles over a smooth projective variety $X$ defined over the field $\mathbb{C}$. When $X$ is a smooth projective surface and $H$ is simple, we construct the algebro-geometric Donaldson-Uhlenbeck compactification of the moduli space of $\mu$-semistable principal $H$-bundles with fixed characteristic classes and describe its points. For large characteristic classes we show that the moduli space of $\mu$-stable principal $H$ bundles is non-empty.
\end{abstract}

\section{Introduction}

The purpose of this paper can broadly be termed two-fold. Its first objective is to prove the semistable reduction theorem for the isomorphism classes of $\mu$-semistable principal bundles (in the sense of RamanathanMumford) with a semisimple structure group $H$ over smooth projective varieties $X$ defined over $\mathbb{C}$. In fact, we prove the semistable reduction theorem for classes of $\mu$-semistable quasibundles (Def. 2.1). This generalises in its entirety the basic theorem of Langton, which proves that the functor of isomorphism classes of $\mu$-semistable torsion-free sheaves is proper. The approach is a generalisation of the one in $[\mathbf{3}]$ and $[4]$, where this theorem is proved for curves.

Carrying out the generalisation to the higher dimensional case involves several new ingredients; for instance, one needs the new notion of quasibundles (due to A. Schmitt). This plays the role of the $\mu$ semistable torsion-free sheaf so as to realise the boundary points. The final proof is concluded with key inputs from Bruhat-Tits theory.

Since the proof of the semistable reduction theorem is rather long and complicated, it is probably appropriate at this point to highlight the basic differences between our proof and that of Langton in the case of families of torsion-free sheaves. Except at the very beginning, our

Received 06/07/2005. 
proof follows an entirely different path than that of Langton primarily because of the fact that it is not even clear if there is a canonical extension of a family of principal bundles parametrised by a punctured disc, be it even unstable, across the puncture. The problem is no longer sheaf theoretic and one is forced to address the problem of torsors with structure group which could be non-reductive group schemes. It is to handle this problem that one needs some aspects of Bruhat-Tits theory. We believe that these new aspects which come up here should also be of general applicability in similar situations where compactification questions need to be addressed for bundles with general structure groups.

More precisely, in [24] Langton first extends the family of semistable torsion free sheaves to a torsion-free sheaf in the limit although nonsemistable. In other words, the "structure group" of the limiting bundle over a big open subset still remains $G L_{n}$. Then by a sequence of Hecke modifications Langton reaches the semistable limit without changing the isomorphism class of the sheaf over the generic fibre. Instead, we are forced to extend the family of semistable rational $H_{K}$-bundles to a rational $H_{A}^{\prime}$-bundle with the limiting bundle remaining semistable, but the structure group becoming non-reductive in the limit (being the closed fibre of the group scheme $H_{A}^{\prime}$ ). In other words, one loses the reductivity of the structure group scheme. Then by using Bruhat-Tits theory, we relate the group scheme $H_{A}^{\prime}$ to the reductive group scheme $H_{A}$ without changing the isomorphism class of the bundle over the generic fibre as well as the semistability of the limiting bundle.

Let $A$ be a complete discrete valuation ring and let $K$ be its quotient field and $k=\mathbb{C}$ its residue field. Our first main theorem is the following ( $X$ is an arbitrary smooth complex projective variety):

Theorem 1.1 (Theorem 4.1). Let $\mathrm{P}_{K}$ be a family of semistable principal $H$-quasibundles on $X \times \operatorname{Spec} K$, or equivalently, if $H_{K}$ denotes the group scheme $H \times \operatorname{Spec} K$, a semi-stable $H_{K}$-quasibundle $\mathrm{P}_{K}$ on $X_{K}$. Then there exists a finite extension $L / K$, with the integral closure $B$ of $A$ in $L$, such that $\mathrm{P}_{K}$, after base change to $\operatorname{Spec} B$, extends to a semistable $H_{B}$-quasibundle $\mathrm{P}_{B}$ on $X_{B}$.

We now turn to the second goal of this paper, which is to give an algebro-geometric compactification of the moduli space of $\mu$-semistable principal bundles over smooth projective surfaces. In fact, we construct a reduced projective scheme which can be termed the DonaldsonUhlenbeck compactification of the moduli space of $\mu$-stable principal $H$-bundles for a general simple group $H$ (cf. Corollary 6.18). In the vector bundle case such an algebro-geometric construction was given by J. Li (cf. [26], [27]). (See also J. Morgan ([30])).

The theorem of Ramanathan and Subramaniam $([\mathbf{3 6}])$, which is a generalisation Donaldson-Uhlenbeck-Yau theorem to the case of principal bundles, gives an identification of antiselfdual (ASD) Yang-Mills 
bundles over $X$ with general structure groups with $\mu$-stable principal bundles. Therefore, our construction of the Donaldson-Uhlenbeck compactification can be viewed as a natural compactification of the moduli space of antiselfdual (ASD) Yang-Mills bundles over $X$ with general structure groups. We remark that for the case of principal bundles with ASD connections, even a topological compactification has not been constructed although one can perhaps extract such a construction from the text $[\mathbf{1 2}]$.

More precisely, we have the following theorem:

Theorem 1.2 (Theorem 6.19).

1) Let $H$ be a semisimple algebraic group and $\rho: H \hookrightarrow S L(V)$ a faithful rational representation of $H$. There exists a reduced projective scheme $M_{H}(\rho)$ which parametrises equivalence classes of $\mu$-semistable $H$-quasibundles with fixed characteristic classes, on the smooth projective surface $X$. This has an open subscheme of equivalence classes of $\mu$-semistable principal $H$-bundles $M_{H}^{0}$.

2) Let $H$ be a simple algebraic group. Then set-theoretically the closure $\overline{M_{H}^{0}}$ of $\mu$-semistable principal $H$-bundles $M_{H}^{0}$ in $M_{H}(\rho)$ is a subset of the disjoint union:

$$
\mathfrak{M}_{H}=\coprod_{l \geq 0} M_{H}^{\mu-\text { poly }}(\mathrm{c}-l) \times S^{l}(X)
$$

where $M_{H}^{\mu-\text { poly }}(\mathrm{c}-l)$ is the moduli space of $\mu$-semistable principal $H$-bundles with characteristic classes $\mathrm{c}-l$ (represented as classes of polystable bundles); in particular, the big stratum is $M_{H}^{\mu-\text { poly }}(\mathrm{c})=M_{H}^{0}$.

3) When $H$ is simple the underlying set of points of the moduli space $\overline{M_{H}^{0}}$, up to homeomorphism, is independent of the representation $\rho$.

4) Let $m=m_{\rho}$ be the Dynkin index of the representation $\rho$ (Def. 6.1). The canonical morphism $f_{\rho}: M_{H}(\rho) \longrightarrow M_{S L(V)}$ maps a copy of $S^{l}(X) \subset M_{h}(\rho)$ to the symmetric power $S^{m l}(X) \subset M_{S L(V)}$ by sending any cycle $Z$ to $m \cdot Z$.

In the above theorem, the formal construction of the moduli space is by itself not too difficult. However, the description of its points is quite involved. The method of proof is along the lines of the proof of J. Li (cf. [26]) and the methods in the paper of Le Potier ([25]) (cf. [21] for a lucid treatment of this approach). In the description of the points of the moduli space of $H$-quasibundles and their relationship with the associated moduli space of $S L(V)$-bundles, the notion of Dynkin index of the representation $\rho$ makes a natural entry and plays a key role in defining intrinsically the cycles associated to the points of the boundary of the moduli space. Its significance has already been noted in the paper 
by Atiyah, Hitchin and Singer [2] for bundles on the real four-sphere and in the paper by Kumar, Narasimhan and Ramanathan $[\mathbf{2 2}]$ for principal bundles on curves.

Since the construction of the Donaldson-Uhlenbeck compactification even in the case of vector bundles $([\mathbf{2 6}])$ is not done entirely by the methods of GIT, it is only natural that for the general case of arbitrary structure groups which we consider, the use of GIT is only peripheral. We may recall that the methods of GIT give as a consequence the projectivity of the quotient space constructed. From this standpoint, our first theorem (Theorem 4.1) is absolutely essential towards proving the compactness. The approach is to separate the proof of the properness (by proving the semistable reduction), and the construction of the moduli (by the process of separating points using sections of a suitable determinant line bundle).

In [11], S. Donaldson remarks that it is natural to expect a generalised theory for his polynomial invariants arising from the Yang-Mills moduli for bundles with general structure groups. He also comments that the Uhlenbeck compactness theorem should naturally hold for the case of general structure groups. One could say that this is indeed the case in the light of the semistable reduction theorem mentioned above as well as the description of the points of the moduli which are added to compactify the ASD moduli space. It is further remarked in [11] that if a general theory of these moduli spaces is given, then one expects results such as the vanishing theorems for these invariants to hold in this general setting. The existence theorem and the description of points of the moduli in the present paper aims at securing the foundations of a precise theory towards this end.

After this paper was completed, the work of Braverman, Finkelberg and Gaitsgory ([9]) was brought to our attention by Alexander Schmitt. They base themselves on a formulation due to V. Ginzburg. The issues which motivate them in considering Uhlenbeck spaces for principal bundles with arbitrary structure groups are deep and far-reaching but quite distinct from ours. Our paper and [9] both aim at the construction of these spaces, but the methods used are also altogether different. The coincidence in terminology of quasibundles is also surprising since their notion and ours do not seem to be related. The method in $[\mathbf{9}]$ can broadly be termed adèlic and in the setting of curves this has been used earlier in $[\mathbf{2 2}]$.

We believe that in this paper we have in fact settled affirmatively some of the basic questions raised in $[\mathbf{9}$, p. 1], particularly those related to moduli. It should be very interesting to establish precise relationships between our paper and [9] (see also Concluding remarks). 
In the final section we prove the following existence theorem on which hinges any computation of Donaldson polynomials associated to these moduli spaces.

Theorem 1.3 (Theorem 7.10). Let $H$ be a semisimple algebraic group over $\mathbb{C}$. Then the moduli space $M_{H}(c)^{s}$ of $\mu$-stable principal $H-$ bundles on a smooth projective surface $X$ is non-empty for large $c$.

In the case when $H=S L(2)$ this is highly non-trivial and uses some deep ideas; this is due to Taubes [43] and later due to Gieseker [17]. Both methods are deformation theoretic, but the method used by Taubes is differential geometric (gluing techniques), whereas Gieseker used degeneration techniques in an algebraic geometric setting to prove the non-emptiness of the moduli space of $\mu$-stable $S L(2)$-bundles. Our approach for the general case of arbitrary semisimple $H$ is to draw on some classical representation theory, by using what are known as principal $S L(2)$ 's in a semisimple group. We then construct $\mu$-stable principal $H$-bundles starting from $S L(2)$-bundles for such principal $S L(2)$ 's in $H$. The important point in these existence results as well as ours is that the bounds are dependent only on $p_{g}(X)$ and not on the polarisation $\Theta$ on $X$. Note that our theorem implies the non-emptiness of $\mu$-stable $S L(r)$-bundles for all $r$.

The proof of this theorem (where we construct $H$-bundles starting from $S L(2)$-bundles with $S L(2) \subset H)$ and the construction of the moduli space, where we use a faithful representation $H \subset S L(V)$, indicate the strong possibility of an algebra of Donaldson polynomials coming from the tensor structure on the category of representations of $H$.

Very recently, Gómez and Sols ([19]) and Schmitt ([37], [38]) have constructed compactifications of moduli spaces of principal bundles on higher dimensional varieties using the Gieseker-Maruyama approach for torsion-free sheaves. The non-emptiness of these spaces (over surfaces) is also therefore a consequence of our Theorem 7.10.

Gómez and Sols follow and generalise the methods of Ramanathan to higher dimensional varieties. Schmitt gives an alternative approach, via GIT again, for the moduli construction but in either case this means that they work with a "Gieseker-Maruyama" type definition for semistability. Schmitt introduces the concept of honest singular principal bundles to recover the boundary points of the moduli space. The singular bundles of Schmitt or equivalently our quasibundles (Def. 2.1) play the key role of giving the boundary points in our moduli space. It seems possible that the moduli spaces that we have constructed can be recovered by a generalised blow-down of the Gómez-Sols moduli, but this needs to be investigated.

In contrast, the striking feature that emerges here is that the underlying set of points of our moduli space (up to homeomorphism) is independent of the choice of a representation of the structure group, 
while the moduli spaces of Gómez-Sols and Schmitt are invariably dependent on the faithful representation chosen. We, however, make no statement on any natural scheme structure on the moduli space. In fact, this is the case even in the usual Donaldson-Uhlenbeck compactification for vector bundles.

The brief layout of the paper is as follows: Sections 1 to 4 are devoted to the proof of the semistable reduction theorem. Section 5 is devoted to the construction of the compactification and Section 6 to the description of its points. Section 7 contains the proof of the non-emptiness of the moduli spaces of stable bundles for large characteristic classes.

1.1. Notations and Conventions. Throughout this paper, unless otherwise stated, we have the following notations and assumptions:

(a) We work over an algebraically closed field $k$ of characteristic zero and without loss of generality we can take $k$ to be the field of complex numbers $\mathbb{C}$.

(b) $X$ will be a smooth projective variety over $k$ till $\S 4$. From $\S 5$ onwards it will be a smooth projective surface.

(c) We fix a hyperplane $\Theta$ on $X$ throughout and will use $\Theta$ for all degree computations.

(d) By a large or big open subset $U \subset X$, we mean a subset such that $\operatorname{codim}_{X}(X-U) \geq 2$.

(e) $H$ is a semisimple algebraic group, and $G$, unless otherwise stated, will always stand for the general linear group $G L(V)$ for a finite dimensional vector space $V$. Their representations are finite dimensional and rational.

(f) $A$ is a discrete valuation ring (which could be assumed to be complete) with residue field $k$, and quotient field $K$.

(g) Let $E$ be a principal $G$-bundle on $X \times T$ where $T$ is Spec $A$. If $x \in X$ is a closed point then we shall denote by $E_{x, A}$ or $E_{x, T}$ (resp $\left.E_{x, K}\right)$ the restriction of $E$ to the subscheme $x \times \operatorname{Spec} A$ or $x \times T$ $(\operatorname{resp} x \times \operatorname{Spec} K)$. Similarly, $p \in T$ will denote the closed point of $T$ and the restriction of $E$ to $X \times p$ will be denoted by $E_{p}$.

(h) In the case of $G=G L(V)$, when we speak of a principal $G$-bundle we identify it often with the associated vector bundle (and can therefore talk of the degree of the principal $G$-bundle with respect to the choice of $\Theta$ ).

(i) We denote by $E_{K}\left(\operatorname{resp} E_{A}\right)$ the principal bundle $E$ on $X \times \operatorname{Spec} K$ (resp $X \times \operatorname{Spec} A$ ) when viewed as a principal $H_{K}$-bundle (resp $H_{A}$-bundle). Here $H_{K}$ and $G_{K}\left(\operatorname{resp} H_{A}\right.$ and $\left.G_{A}\right)$ are the product group schemes $H \times \operatorname{Spec} K$ and $G \times \operatorname{Spec} K(\operatorname{resp} H \times \operatorname{Spec} A$ and $G \times \operatorname{Spec} A)$.

(j) If $H_{A}$ is an $A$-group scheme, then by $H_{A}(A)\left(\operatorname{resp} H_{K}(K)\right)$ we mean its $A(\operatorname{resp} K)$-valued points. When $H_{A}=H \times \operatorname{Spec} A$, 
then we simply write $H(A)$ for its $A$-valued points. We denote the closed fibre of the group scheme by $H_{k}$.

(k) Let $Y$ be any $G$-variety and let $E$ be a $G$-principal bundle. For example $Y$ could be a $G$-module. Then we denote by $E(Y)$ the associated bundle with fibre type $Y$ which is the following object: $E(Y)=(E \times Y) / G$ for the twisted action of $G$ on $E \times Y$ given by $g .(e, y)=\left(e . g, g^{-1} \cdot y\right)$.

(l) If we have a group scheme $H_{A}\left(\operatorname{resp} H_{K}\right)$ over $\operatorname{Spec} A(\operatorname{resp} \operatorname{Spec} K)$ an $H_{A}$-module $Y_{A}$ and a principal $H_{A}$-bundle $E_{A}$. Then we shall denote the associated bundle with fibre type $Y_{A}$ by $E_{A}\left(Y_{A}\right)$.

(m) By a family of $H$ bundles on $X$ parametrised by $T$ we mean a principal $H$-bundle on $X \times T$, which we also denote by $\left\{E_{t}\right\}_{t \in T}$.

Acknowledgments. I am grateful to C.S. Seshadri and D.S. Nagaraj for their patience and help in this work. I thank M.S. Narasimhan, V. Uma and S. Bandhopadhaya for helpful discussions. I am extremely grateful to Alexander Schmitt for a meticulous reading of an earlier version of this paper. His comments have been very helpful in clarifying many issues in this paper.

\section{Rational bundles and principal quasibundles}

Let $X$ be a smooth projective variety over $\mathbb{C}$. Let $\rho: H \hookrightarrow S L(V)$. Let $\mathcal{E}$ be a torsion-free sheaf on $X$ and let $U(\mathcal{E})$ be the largest open subset where $\mathcal{E}$ is locally free. Let $S_{\mathcal{E}}$ be the affine $X$-scheme given by $\operatorname{Spec} \operatorname{Sym}^{*}(\mathcal{E} \otimes \mathcal{O}(V))$. There is a canonical action of $H$ on $S_{\mathcal{E}}$ and we consider the categorical quotient $\operatorname{Spec}\left(\operatorname{Sym}^{*}(\mathcal{E} \otimes \mathcal{O}(V))^{H}\right)=$ $\left(S_{\mathcal{E}}\right) / / H$. Since $H$ is assumed to be semisimple, the action has a nonempty collection of semistable points.

Definition 2.1 (Rational principal bundles).

(a) Following $([\mathbf{3 3}])$, by a rational principal $H$-bundle, we mean a principal $H$-bundle on a large open $U \subset X$.

Definition 2.2 (Principal quasibundles). We fix the following data (cf $[37])$ :

(a) A faithful representation $H \hookrightarrow S L(V)$.

(b) A torsion-free sheaf $\mathcal{E}$ with generic fibre of type $V^{*}$ and trivial determinant.

(c) Let $U(\mathcal{E})$ be the maximal open subset where $\mathcal{E}$ is locally free. Suppose further that we are given a morphism (which we term a reduction section for the obvious reasons) $\sigma: X \longrightarrow\left(S_{\mathcal{E}}\right) / / H$, which on the open subset $U(\mathcal{E})$ gives a genuine reduction of structure group to $H$.

(d) We obtain an $X$-scheme $\mathrm{P} \longrightarrow X$, by pulling back the quotient map $q: S_{\mathcal{E}} \longrightarrow\left(S_{\mathcal{E}}\right) / / H$ and the following diagram: 


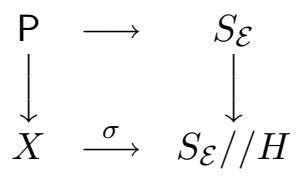

By an $H$-quasibundle, we mean a scheme $\mathrm{P} \longrightarrow X$ as above. Equivalently, an $H$-quasibundle is given by a pair $(\mathcal{E}, \sigma)$ which may be considered as a datum for a generalised reduction of structure group.

Remark 2.3. This notion is due to Alexander Schmitt, and the pair $(\mathcal{E}, \sigma)$ is termed by him an honest singular principal bundle. This is a natural generalisation of the classical notion of a frame bundle associated to a vector bundle.

Remark 2.4. Let the open subset $U(\mathrm{P})=U(\mathcal{E})$ denote the largest open subset of $X$ where $\mathrm{P}$ is a genuine principal $H$-bundle. In particular, $\left.\mathrm{P}\right|_{U(\mathrm{P})}$ is a rational principal $H$-bundle.

Remark 2.5. Since the notion of quasibundles will play a key role in what proceeds, we will briefly recall its salient features, especially those which will be frequently used in this paper.

Let $T$ be an arbitrary normal variety and $\mathcal{E}$ a torsion-free sheaf on $T$. We can identify the affine $T$-scheme $S_{\mathcal{E}}$ with the space $\underline{\text { Hom }}(\mathcal{E}$, $\left.V^{*} \otimes \mathcal{O}_{X}\right)$ and similarly the affine $T$-scheme $S_{\mathcal{E}} / / H$ with the space $\underline{\text { Hom }}\left(\mathcal{E}, V^{*} \otimes \mathcal{O}_{X}\right) / / H$ (cf. $[37,3.7,3.8]$ ).

Let $\mathcal{U}$ be the maximal open subset of $T$ such that $\left.\mathcal{E}\right|_{\mathcal{U}}$ is locally free with general fibre $V$ and trivial determinant and let $\rho: H \hookrightarrow G L(V)$. Then one knows that $\mathcal{U}$ is a big open subset of $T$. A reduction of structure group of the principal $G L(V)$-bundle underlying the vector bundle $\left.\mathcal{E}\right|_{\mathcal{U}}$ can be viewed as a section:

$$
\sigma_{\mathcal{U}}: \mathcal{U} \longrightarrow \underline{\operatorname{Isom}}\left(\left.\mathcal{E}\right|_{\mathcal{U}}, V \otimes \mathcal{O}_{X}\right) / H .
$$

Now observe that $\underline{\operatorname{Isom}}\left(\left.\mathcal{E}\right|_{\mathcal{U}}, V \otimes \mathcal{O}_{X}\right) / H \subset S_{\mathcal{E}} / / H$ and further, $S_{\mathcal{E}} / / H$, can be embedded as closed subscheme of a vector bundle over $T$. (This follows for example by [41, Theorem 3 and Remark 10], where the finite generation of invariants is shown over any base scheme. We remark that in our case, since we work only over characteristic zero, this also follows as in the classical setting since the Reynolds operator can be defined over $T$.)

It therefore follows by Hartogs theorem that the reduction $\sigma_{\mathcal{U}}$ extends uniquely to a section:

$$
\sigma: T \longrightarrow S_{\mathcal{E}} / / H
$$

Following [35] we have the following definitions.

Definition 2.6 (A. Ramanathan). A rational principal $H$-bundle $E$ is said to be $\mu$-semistable (resp. $\mu$-stable) if $\forall$ parabolic subgroup $Q$ of $H, \forall$ reduction $\sigma_{Q}: X \longrightarrow \mathrm{P}(H / Q)$ and $\forall$ dominant character $\chi$ 
of $Q$, the bundle $\left.\sigma_{Q}^{*}\left(L_{\chi}\right)\right)$ has degree $\leq 0($ resp. $<0)($ cf. $[35])$. We note that in this convention, a dominant character $\chi$ of $Q$ induces a negative ample line bundle on $H / Q$. Note further that this definition makes sense since the degree of the line bundle is well defined on large open subsets. This definition works for reductive groups as well.

Definition 2.7. A $H$-quasibundle $\mathrm{P}$ is said to be $\mu$-semistable (resp. $\mu$-stable) if the induced rational principal $H$-bundle $\left.\mathrm{P}\right|_{U(\mathrm{P})}$ is $\mu-s e m i$ stable (resp. $\mu$-stable).

Definition 2.8. A reduction of structure group of $E$ to a parabolic subgroup $Q$ is called admissible if for any character $\chi$ on $Q$ which is trivial on the center of $H$, the line bundle associated to the $Q$-bundle $E_{Q}$ obtained by the reduction of structure group has degree zero.

Definition 2.9. An $H$-bundle $E$ is said to be polystable if it has a reduction of structure group to a Levi subgroup $R$ of a parabolic $Q$ such that the $R$-bundle $E_{R}$ obtained by the reduction is stable and the extended $Q$ bundle $E_{R}(Q)$ is an admissible reduction of structure group for $E$. Since the definition involves only degrees of line bundles, it clearly holds good for rational principal bundles as well (cf. [33]).

Remark 2.10. It is clear that we have the natural notions of polystability for $H$-quasibundles as well. So a quasibundle $\mathrm{P}$ is termed polystable if the induced rational principal $H$-bundle $\left.\mathrm{P}\right|_{U(\mathrm{P})}$ is polystable.

2.0.1. Semistability and polystability over curves. The study of semistability and polystability of principal bundles on curves was initiated by A.Ramanathan. Over the years it has developed in many directions and the results which one requires are scattered in the literature. Polystability is also differently called quasi-stability in the literature but we avoid this terminology for obvious reasons (we have already a notion of quasibundles ..).

For the convenience of the reader we gather some of the relevant facts with appropriate references in the following theorem:

Theorem 2.11. Let $C$ be a smooth projective curve over the field of characteristic zero. Let $H$ a semisimple algebraic group.

The following are equivalent:

(i) A principal $H$-bundle $E$ is polystable in the sense of Ramanathan (Def. 2.9).

(ii) There exists a faithful representation $H \hookrightarrow G L(V)$ such that the associated bundle $E(V)$ is polystable of degree 0 .

(iii) For every representation $H \longrightarrow G L(W)$, the bundle $E(W)$ is polystable of degree 0.

(iv) Let ad $: H \longrightarrow G L(\mathfrak{H})$ be the adjoint representation. Then $E(\mathfrak{H})$ is polystable (of degree zero). 
(v) The bundle $E$ arises from a representation $\rho: \pi_{1}(C) \longrightarrow K$, where $K$ is a maximal compact subgroup of $H$.

(vi) The bundle E carries an Einstein-Hermitian connection.

Proof. The equivalence of (i) and (v) is the main theorem of Ramanathan, which generalises the Narasimhan-Seshadri theorem for principal bundles. ([34]).

(i) $\Leftrightarrow$ (vi) is the main theorem of [36]. The equivalence (iv) $\Leftrightarrow(\mathrm{v})$ is shown in [1, Lemma 10.12].

By [36, Prop. 1], we may go modulo the center and assume that the group $H$ has trivial center. Therefore, the adjoint representation $a d: H \hookrightarrow G L(\mathfrak{H})$ is a faithful representation. From this standpoint, (ii), (iii) and (iv) are equivalent by a Tannakian principle and the proof can be found in [3, Prop. 2.3]. The argument there is for semistability, but the changes needed to be made for polystability are easy since all bundles involved are of degree 0 (see also the proof of Prop. 3.11 below).

q.e.d.

Remark 2.12. In fact, the equivalence [iv] $\Leftrightarrow[\mathrm{v}]$ holds more generally even for $H$ reductive (cf. [1, Lemma 10.12]).

2.1. Some key lemmas. We need the following couple of lemmas about families of locally-free sheaves which we will use in this work.

Let $C \subset U$ be a smooth projective curve. We recall:

Lemma 2.13 (cf. [3, Lemma 2.10]). Let $T=\operatorname{Spec} A$ and let $E_{T}$ be a family of vector bundles on $C \times T$ such that $E_{p}$ is semistable of degree 0 . Let $s_{K}$ be a section of the family $E_{K}$ restricted to $C \times \operatorname{Spec} K$, with the property that for a base point $x \in C$, the section $s_{K}$ extends along $x \times T$ to give a section of $E_{x \times T}$. Then the section extends to the whole of $C \times T$.

We have more generally:

Lemma 2.14. Let $W$ be a family of semistable vector bundles with $c_{1}=0$ on $U$ parametrised by $T$, i.e., a vector bundle on $U_{A}$, where $U \subset X$ is a large open subset of $X$. Suppose that we are given a regular section $s_{K}: U_{K} \longrightarrow W_{K}$ such that, for an irreducible smooth divisor $Y \subset U$, the section extends as a regular section along $Y_{A}$. Then the section $s_{K}$ extends to a regular section $s_{A}$ on $U_{A}$.

Proof. For the section $s_{T-p}$, viewed as a section of $W_{T-p}$ we have two possibilities, since $U_{A}$ is normal and the polar set is a divisor:

(a) it either extends as a regular section $s_{T}$.

(b) or it has a pole along $U \times p$.

By the given property, we have a section $\sigma_{Y}: Y \times T \longrightarrow W_{Y}$ with the property that, at $\forall x \in Y, s_{t}(x)=\sigma_{Y}(x, t) \forall t \in T-p$; 
So to complete the proof, we need to check that the possibility (b) cannot hold:

Suppose it does hold. Then the section $s_{T-p}=s_{K}$ is a section of $W_{K}$, i.e., a rational section of $W$ with a pole along the divisor $U \times p \subset U \times T$, of order $k \geq 1$.

Thus by multiplying $s_{T-p}$ by $\pi^{k}$ we get a regular section $s_{T}^{\prime}$ of $W$ on $U \times T$. If $s_{T}^{\prime}=\left\{s_{t}^{\prime}\right\}_{t \in T}$, then we have:

(i) $s_{t}^{\prime}=\lambda(t) \cdot s_{t}, t \in T-p$ where $\lambda: T \longrightarrow \mathbb{C}$ is a function given by $\pi^{k}$, having zeros of order $k$ at $p$.

(ii) $s_{p}^{\prime}$ is a non-zero section of $W_{p}$. Here we first note that $W_{p}$ is a bundle on $U$ which is large and hence the $\mu$-semistability is completely determined by $W_{p}$. Further, by taking $W_{p}^{* *}$, this extends as a reflexive sheaf to the whole variety $X$ and remains $\mu$-semistable of degree 0. By Hartogs' theorem (since reflexive sheaves are nor$m a l)$, the section $s_{p}^{\prime}$ extends to a non-zero section of $W_{p}^{* *}$ to the whole of $X$.

Since $\mathcal{O}_{X}$ is stable and degree 0 , it follows that $s_{p}^{\prime}$ gives a short exact sequence of sheaves on $X$ :

$$
0 \longrightarrow \mathcal{O}_{X} \longrightarrow W_{p}^{* *} \longrightarrow Q \longrightarrow 0
$$

where the torsion part of $Q$ is supported in a subset of codimension $\geq 2$ (if the quotient sheaf had a divisor in its support then by taking determinants, we see that there is a contradiction to the equality of the degrees $\left.\operatorname{deg}\left(\mathcal{O}_{X}\right)=\operatorname{deg}\left(W_{p}^{* *}\right)=0\right)$.

Thus it follows that there exists

$$
y \in Y \text { such that } s_{p}^{\prime}(y) \neq 0 .
$$

By the assumption that the section extends along $Y_{A}$, and by the fact that $y \in Y$ it follows that $s_{t}(y)=\sigma_{Y}(y, t)$, for $t \in T-p$, and hence

$$
s_{t}^{\prime}(y)=\lambda(t) \cdot \sigma_{Y}(y, t) \text { for } t \in T-p .
$$

Therefore, by continuity, since $\sigma_{Y}(y, p)$ is well-defined, we see that $\lambda(t)$. $\sigma_{Y}(y, t)$ tends to $\lambda(p) \cdot \sigma_{Y}(y, p)=0$, as $t \longrightarrow p$.

Also, $s_{t}^{\prime}(y) \longrightarrow s_{p}^{\prime}(y)$ since $t \longrightarrow p$. Hence by continuity again, it follows that $s_{p}^{\prime}(y)=0$, which contradicts $(*)$.

Thus the possibility (b) does not occur and we are done. q.e.d.

2.1.1. A Refinement of Langton's theorem. Let $R^{\mu s s}$ denote the subset of the usual Quot scheme which parametrises the usual torsionfree sheaves with fixed topological type and Hilbert polynomial. Let $\mathcal{G}$ be the group $S L(\chi)$ ( $\chi$ being the Euler characteristics of sheaves in $R^{\mu s s}$ ) acting on the (open subset) $R^{\mu s s}$ of the Quot scheme.

Lemma 2.15 (Modified Langton). Let $E_{K}$ denote a family of $\mu-$ semistable torsion-free sheaves of degree zero on $X \times \operatorname{Spec} K$. Then, (by going to a finite cover $S$ of $T$ if need be) the sheaf $E_{K}$ extends (up 
to isomorphism) to a family $E_{A}$ with the property that the limiting sheaf $E_{0}$ is in fact polystable.

Proof. By definition $E_{K}$ gives a $K$-valued point $x_{K}: \operatorname{Spec}(K) \longrightarrow$ $R^{\mu s s}$

By Langton, there exists an $A$-valued point $x_{A}: \operatorname{Spec}(A) \longrightarrow R^{\mu s s}$ such that $x_{p}$ is given by $E_{p}$, which is a semistable torsion-free sheaf.

One knows that (for a choice of a Jordan-Holder filtration) there exists a family $\left.F_{t}\right|_{t \in \mathbb{A}}$ such that $F_{t} \simeq E_{p}$ for $t \neq 0$ and the limit is a polystable sheaf $F_{0} \simeq g r^{\mu}\left(E_{p}\right)$. Let us denote this family by a morphism $f: \mathbb{A} \longrightarrow R^{\mu s s}$ and the point $f(0)=y_{0}$ for $t=0$.

By going to a finite cover if need be, we may assume that there exists a point $g \in \mathcal{G}(\mathbb{C}(t))$ such that $g \cdot E_{p} \simeq F_{\mathbb{C}(t)}$ with limit given by $F_{0}$.

Let $D=\overline{\mathcal{G} \cdot x_{K}}$ (the $\mathcal{G}(K)$-orbit closure in $R^{\mu s s}$ ) be considered as a $\mathbb{C}$-variety. Then by definition the point $x_{p} \in D$ (where $x_{p}$ corresponds to the sheaf $\left.E_{p}\right)$. Further, $D$ is $\mathcal{G}$-stable. Therefore, $g \cdot x_{p} \in D(\mathbb{C}(t))$. Since $D$ is closed it follows that the point $y_{0}$ belongs to $D$.

Again, since $D$ is irreducible, we can join $y_{0}$ to the orbit $\mathcal{G} \cdot x_{K}$ and we get a scheme $S$, the spectrum of a discrete valuation ring, such that $s_{0}=y_{0}$ and $S-s_{0}=\operatorname{Spec}(L)$ where $L$ is a finite extension of $K$ (consider the natural map $\left.q: \mathcal{G} \cdot x_{K} \longrightarrow \operatorname{Spec}(K)\right)$. Therefore $\left(S \cap \mathcal{G} \cdot x_{K}\right)$ is mapped to $\operatorname{Spec}(K)$ by $q$ which gives the extension $L / K$.

We also get a resulting family $\left\{E_{s}^{\prime}\right\}_{s \in S}$ such that $E_{s}^{\prime} \simeq E_{q(s)}$ for $s \neq 0$ and $E_{s_{0}}^{\prime} \simeq g r^{\mu}\left(E_{p}\right)$. We are done.

q.e.d.

\section{Extension of structure group to the flat closure}

Fix a faithful representation $\rho: H \hookrightarrow G$ defined over $\mathbb{C}$. Consider the extension of structure group of the principal $H_{K}$-quasibundle $\left(E_{K}, \sigma_{K}\right)$ via the induced $K$-inclusion $\rho_{K}: H_{K} \hookrightarrow G_{K}$. In other words, on a large open $U_{K}$, we are given a reduction of structure group of the principal $G_{K}$-bundle $\left.E_{K}\right|_{U_{K}}$ to $H_{K}$.

Then, since $G=G L(n)$, by the properness of the functor of semistable torsion-free sheaves (the Theorem of Langton), there exists a semistable extension of $E_{K}$ to a torsion-free sheaf on $X \times \operatorname{Spec} A$, which we denote by $E_{A}$. Call the restriction of $E_{A}$ to $X \times p$ (identified with $X$ ) the limiting bundle of $E_{A}$ and denote it by $E_{p}$ (as in $\S 1$ ). One has in fact slightly more, which is what we need.

3.1. Mehta-Ramanathan restriction theorems. Fix an integer $c \in$ $[1, d-1]$ where $d=\operatorname{dim}(X)$ and

$$
\left(a_{1}, \ldots, a_{c}\right) \in \mathbb{Z}^{c}
$$

with $a_{i} \geq 2$ for all $i \in[1, c]$. For any integer $m \geq 1$, by a complete intersection of type $(m)$ we mean a complete intersection of divisors:

$$
D_{1} \cap D_{2} \cap \cdots \cap D_{c}
$$


with $D_{i} \in\left|a_{i}^{m} \Theta\right|$.

By a general complete intersection subvariety of type $(m)$ we mean the complete intersection formed by the $D_{j}^{\prime} s$ from a non-empty open subset $S$ of the linear system $\prod_{i=1}^{n-1}\left|a_{i}^{m} \Theta\right|$.

Note that for $m \gg 0$, a general complete intersection of type $(m)$ is a smooth irreducible subvariety of $X$ of dimension $d-c$.

We recall the main theorem of $[\mathbf{2 9}]$ :

Theorem 3.1 (Mehta-Ramanathan). Let $V$ be a semi-stable (resp. stable, polystable) rational vector bundle over $U \subset X$. Then for all $m \gg 0$ the restriction $\left.V\right|_{Y^{\circ}}$ to $Y \cap U$, for a general complete intersection $Y$ of type $(m)$, is a semi-stable (resp. stable, polystable) rational vector bundle. Conversely, if $\left.V\right|_{C}$ to a general complete intersection curve of type $(m)$ is semi-stable (resp. stable, polystable) then so is $V$.

Remark 3.2. Consider the extended sheaf $E_{A}$ obtained above with the property that $E_{p}$ is a polystable torsion-free sheaf of degree 0 . Since $E_{p}$ is a polystable torsion-free sheaf of degree 0, let $U=U\left(E_{p}\right) \subset X$ be the largest open set where it is locally free. By the Restriction theorem of Mehta-Ramanathan, for a large $m$, there is an open subset $S \subset$ $\prod_{i=1}^{n-1}\left|a_{i}^{m} \Theta\right|$, such that if $Y \in S$ is a complete intersection subvariety, then $\left.E_{p}\right|_{Y \cap U}$ remains polystable of degree 0 .

Let the ' denote intersection with $U$. We can therefore chose a chain of subvarieties:

$$
C \subset \cdots\left(D_{1} \cap D_{2} \cap \cdots \cap D_{c}\right)^{\prime} \cdots \subset D_{1}^{\prime} \subset U
$$

and the point $x \in C$ such that the restriction $\left.E_{p}\right|_{\left(D_{1} \cap D_{2} \cap \cdots \cap D_{c}\right)^{\prime}}$ for every $c$ is locally free and remains polystable of degree 0 .

Since $A$ is a discrete valuation ring, if we choose an open subset $U \subset X$ where the limiting sheaf $E_{p}$ is locally free, then the family $E_{A}$ is also locally free when restricted to the large open subscheme $U_{A}$ (this is easy to see. cf. for example Lemma $5.4[\mathbf{3 2}]$ ). Hence, if we further restrict $E_{A}$ to the curve $C_{A} \subset U_{A}$, we get a family of locally free sheaves $\left.E_{A}\right|_{C_{A}}$ on the smooth projective curve $C$ parametrized by SpecA. Further, by choice, the limiting bundle $E_{p}$ is polystable on $C$ of degree 0 .

3.2. The Flat closure. We observe the following:

- Note that giving a reduction of structure group of the $G_{K}$-bundle $E_{K}$ on a large $U_{K}$ is equivalent to giving a section $s_{K}$ of $E_{K}\left(G_{K} /\right.$ $\left.H_{K}\right)$ over $U_{K}$.

- Let

$$
C \subset \cdots\left(D_{1} \cap D_{2} \cap \cdots \cap D_{c}\right)^{\prime} \cdots \subset D_{1}^{\prime} \subset U=U\left(E_{p}\right)
$$

be as in (3.2) above. We fix a base point $x \in C \subset U$ and denote by $x_{A}=x \times \operatorname{Spec} A$, the induced section of the family (which we 
call the base section):

$$
X_{A} \longrightarrow \operatorname{Spec} A .
$$

Since $x \in U$, it allows us to work with genuine principal bundles and their restrictions to the section $x_{A}$ defined by the base point $x$.

- From now on, unless otherwise stated, we shall fix this chain of smooth subvarieties of $U$ along with the base point $x$.

- Let $E_{x, A}\left(\operatorname{resp} E_{x, K}\right)$ be as in $\S 1$, the restriction of $E_{A}$ to $x_{A}$ (resp $\left.x_{K}\right)$. Thus $s_{K}(x)$ is a section of $E_{K}\left(G_{K} / H_{K}\right)_{x}$ which we denote by $E_{x}\left(G_{K} / H_{K}\right)$.

- Since $E_{x, A}$ is a principal $G$-bundle on Spec $A$ and therefore trivial, it can be identified with the group scheme $G_{A}$ itself. For the rest of the article we fix one such identification, namely:

$$
\xi_{A}: E_{x, A} \longrightarrow G_{A} .
$$

- Since we have fixed $\xi_{A}$ we have a canonical identification

$$
E_{x}\left(G_{K} / H_{K}\right) \simeq G_{K} / H_{K}
$$

which therefore carries a natural identity section $e_{K}$ (i.e., the coset $\left.i d . H_{K}\right)$. Using this identification we can view $s_{K}(x)$ as an element in the homogeneous space $G_{K} / H_{K}$.

- Let $\theta_{K} \in G(K)$ be such that $\theta_{K}^{-1} \cdot s_{K}(x)=e_{K}$. Then we observe that the isotropy subgroup scheme in $G_{K}$ of the section $s_{K}(x)$ is $\theta_{K} \cdot H_{K} \cdot \theta_{K}^{-1}$. (We remark that such a $\theta_{K}$ will exist after going to a finite extension of $K$. By an abuse of notation we will continue calling this extension as $K$. This is required since $G_{K} \longrightarrow G_{K} / H_{K}$ need not be locally trivial.)

- On the other hand one can realize $s_{K}(x)$ as the identity coset of $\theta_{K} \cdot H_{K} \cdot \theta_{K}^{-1}$ by using the following identification:

$$
\begin{aligned}
G_{K} / \theta_{K} \cdot H_{K} \cdot \theta_{K}^{-1} \stackrel{\sim}{\longrightarrow} G_{K} / H_{K} . \\
g_{K}\left(\theta_{K} \cdot H_{K} \cdot \theta_{K}^{-1}\right) \longmapsto g_{K} \theta_{K} \cdot H_{K} .
\end{aligned}
$$

Definition 3.3. Let $H_{K}^{\prime}$ be the subgroup scheme of $G_{K}$ defined as:

$$
H_{K}^{\prime}:=\theta_{K} \cdot H_{K} \cdot \theta_{K}^{-1} \cdot
$$

Using $\xi_{A}$ we can have a canonical identification:

$$
E_{x}\left(G_{K} / H_{K}^{\prime}\right) \simeq G_{K} / H_{K}^{\prime}
$$

Then we observe that, using the above identification, we get a section $s_{K}^{\prime}$ of $E_{K}\left(G_{K} / H_{K}^{\prime}\right)$ with the property that $s_{K}^{\prime}(x)$ is the identity section, and, moreover, since we have conjugated by an element $\theta_{K} \in G_{A}(K)(=$ $G(K))$, the isomorphism class of the $H_{K}$-bundle $P_{K}$ given by $s_{K}$ does not change by going to $s_{K}^{\prime}$. 
Thus in conclusion, the $G_{A}$-bundle $E_{A}$ has a reduction to $H_{K}^{\prime}$ given by a section $s_{K}^{\prime}$ of $E_{K}\left(G_{K} / H_{K}^{\prime}\right)$, with the property that, at the given base section $x_{A}=x \times \operatorname{Spec} A$, we have an equality $s_{K}^{\prime}\left(x_{A}\right)=e_{K}^{\prime}$, with the identity element of $G_{K} / H_{K}^{\prime}$ (namely the coset $i d . H_{K}^{\prime}$ ).

Definition 3.4. The flat closure of the reduced group scheme $H_{K}^{\prime}$ in $G_{A}$ is defined to be the schematic closure of $H_{K}^{\prime}$ in $G_{A}$ with the reduced scheme structure. Let $H_{A}^{\prime}$ denote the flat-closure of $H_{K}^{\prime}$ in $G_{A}$.

Remark 3.5. Let $I_{K}$ be the ideal defining the subgroup scheme $H_{K}^{\prime}$ in $K(G)$ (note that $G_{A}\left(\operatorname{resp} G_{K}\right)$ is an affine group scheme and we denote by $A(G)$ (resp $K(G))$ its coordinate ring). If we set $I_{A}=$ $I_{K} \cap A(G)$, then it is easy to see that since we are over a discrete valuation ring, $I_{A}$ is in fact the ideal in $A(G)$ defining the flat closure $H_{A}^{\prime}$.

We then have a canonical identification via $\xi_{A}$ :

$$
E_{x}\left(G_{A} / H_{A}^{\prime}\right) \simeq G_{A} / H_{A}^{\prime} .
$$

One can easily check that $H_{A}^{\prime}$ is indeed a subgroup scheme of $G_{A}$ since it contains the identity section of $G_{A}$, and moreover, it is faithfully flat over $A$. Notice however that $H_{A}^{\prime}$ need not be a reductive group scheme; that is, the special fibre $H_{k}$ over the closed point need not be reductive.

Observe further that $s_{K}^{\prime}(x)$ extends in a trivial fashion to a section $s_{A}^{\prime}(x)$, namely the identity coset section $e_{A}^{\prime}$ of $E_{x}\left(G_{A} / H_{A}^{\prime}\right)$ identified with $G_{A} / H_{A}^{\prime}$.

3.3. Chevalley embedding of $G_{A} / H_{A}^{\prime}$. As we have noted, $H_{A}^{\prime}$ need not be reductive and the rest of the proof consists of getting around this difficulty. Our first aim is to prove that the structure group of the bundle $E_{A}\left(G_{A}\right)$ can be reduced to $H_{A}^{\prime}$, which is the statement of Proposition 3.7.

We need the following generalisation of a well-known result of Chevalley from $[\mathbf{3}]$.

Lemma 3.6. There exists a finite dimensional $G_{A}$-module $W_{A}$ such that $G_{A} / H_{A}^{\prime} \hookrightarrow W_{A}$ is a $G_{A}$-immersion.

3.4. Extension to flat closure and local constancy. Recall that the section $s_{K}^{\prime}(x)$ extends along the base section $x_{A}$, to give $s_{A}^{\prime}(x)=w_{A}$. The aim of this section is to prove the following key theorem.

Theorem 3.7. There exists a large open subset $U^{o} \subset U$ such that the section $s_{K}^{\prime}$ extends to a section $s_{A}^{\prime}$ of $E_{A}\left(G_{A} / H_{A}^{\prime}\right)$ when we restrict $E_{A}$ to the open subset $U_{A}^{o} \subset U_{A}$. In other words, the structure group of $E_{\left.A\right|_{U_{A}}}$ can be reduced to $H_{A}^{\prime}$; in particular, if $H_{k}^{\prime}$ denotes the closed fibre of $H_{A}^{\prime}$, then the structure group of $E_{k}$ can be reduced to $H_{k}^{\prime}$.

Towards this we need the following key result. 
Proposition 3.8. Let $E$ be a polystable principal $G$-bundle of degree zero on a smooth projective curve $C$ (here $G=S L(n)$ or $G L(n))$. Let $W$ be a $G$-module and $N$ a $G$-subscheme of $W$ of the form $G / H^{\prime}$.

If $s$ is a section of $E(W)$ such that for some $x \in C, s(x)$ lies in the fibre $E(N)_{x}$ of the fibration $E(N)$ at $x \in C$, then the entire image of $s$ lies in $E(N)$. Consequently, $E$ has a reduction of structure group to the subgroup $H^{\prime}$.

Proof. The bundle $E$ being polystable, it is defined by a "unitary" representation

$$
\chi: \pi_{1}(C) \longrightarrow G
$$

which maps into a maximal compact subgroup of $G$. This implies that if the universal covering $j: Z \longrightarrow C$ is considered as a principal fibre space with structure group $\pi_{1}(C)$, then the principal $G$-bundle $E$ is the associated bundle through $\chi$.

Let $\rho: G \longrightarrow G L(W)$ be the representation defining the $G$-module $W$. Then $E(W)$ can be considered as the bundle associated to the principal bundle $j: Z \longrightarrow C$ through the representation

$$
\rho \circ \chi: \pi_{1}(C) \longrightarrow G L(W),
$$

which maps into the unitary subgroup of $G L(W)$.

By generalities on principal bundles and associated constructions, since

$$
E(W) \simeq Z \times{ }^{\pi_{1}(C)} W,
$$

a section of $E(W)$ can be viewed as a $\pi_{1}(C)$-map

$$
s_{1}: Z \longrightarrow W \text {. }
$$

Now, since $Z$ is the universal cover of the curve $C$ and $s$ is a section of $E(W)$, one knows (cf. $[\mathbf{3 1}]$ ) that there exists a $\pi_{1}(C)$-invariant element $w \in W$ such that $s$ is defined by a map

$$
s_{1}: Z \longrightarrow W
$$

given by $s_{1}(x)=w, \forall x \in C$, i.e., "the constant map sending everything to $w "$.

Since $w \in W$ is a $\pi_{1}(C)$-invariant vector and the action of $\pi_{1}(C)$ is via the representation $\chi$, we see that $\chi$ factors via

$$
\chi_{1}: \pi_{1}(C) \longrightarrow H^{\prime}
$$

since $H^{\prime}=\operatorname{Stab}_{G}(w)$.

In particular, we get the $H^{\prime}$-bundle from the representation $\chi_{1}$ and clearly this $H^{\prime}$-bundle is the reduction of structure group of the $G$ bundle $E$ given by the section $s$.

By the very construction of the reduction, the induced $H^{\prime}$-bundle is flat and also semistable since it comes as the reduction of the structure group of the polystable bundle $E$ (by Def. 3.9 below). This proves Proposition 3.8. 
Definition 3.9. Let $H^{\prime}$ be an affine algebraic group not necessarily reductive. Let $P$ be a principal $H^{\prime}$-bundle on $C$ a smooth projective curve. We define $P$ to be polystable if:

1) It is flat (in the sense that it comes from the representation $\chi$ of the fundamental group of $C$ );

2) there exists a faithful representation

$$
\rho: H^{\prime} \longrightarrow G L(V)
$$

such that the associated vector bundle $P(V)$ is polystable of degree zero.

Remark 3.10. This definition is ad hoc and made only to suit our needs. Let $\mathcal{M}:=\mathcal{M}(\rho \circ \chi)$ denote the Zariski closure of the image of $\rho \circ \chi: \pi_{1}(C) \longrightarrow G L(V)$ in $G L(V)$. We term this the "monodromy" subgroup associated to the representation $\rho \circ \chi$.

Since the bundle associated to $\rho \circ \chi: \pi_{1}(C) \longrightarrow G L(V)$ i.e., $P(V)$, is assumed to be polystable of degree 0 , by the Narasimhan-Seshadri theorem, the representation $\rho \circ \chi$ is unitary and also the monodromy subgroup $\mathcal{M}$ is reductive (possibly non-connected). This can be viewed as a Tannakian interpretation of polystability. Further, since $\chi$ maps into $H^{\prime}$, the monodromy subgroup $\mathcal{M}$ is a subgroup of $H^{\prime}$.

Let us denote the inclusion of the monodromy subgroup by:

$$
\iota: \mathcal{M} \hookrightarrow H^{\prime} .
$$

The fact that the bundle $P$ comes from an associated construction via the homomorphism $\chi$ implies that $P$ has a reduction of structure group to $\mathcal{M}$. Let the resulting $\mathcal{M}$ bundle be denoted by $P_{\mathcal{M}}$.

Proposition 3.11. Let $H^{\prime}$ be an affine algebraic group (not necessarily reductive), as above, and let $P$ be a polystable principal $H^{\prime}$-bundle on a smooth projective curve $C$. Let $f: H^{\prime} \longrightarrow H$ be a morphism from $H^{\prime}$ to a semisimple group $H$. Then the associated principal $H$-bundle $P(H)$ is also polystable.

Proof. Since $P$ has a reduction of structure group to $\mathcal{M}$, we may view the principal $H$ bundle $P(H)$ as obtained from the homomorphism $f \circ \iota: \mathcal{M} \longrightarrow H$. Thus $P(H)$ can be identified with $P_{\mathcal{M}}(H)$.

To check the polystability of the principal $H$-bundle $P(H)$ by Theorem 2.11, we need only check that if $\psi: H \longrightarrow G L(W)$ is any representation of $H$ then the associated bundle $P(H)(W)$ is polystable. In other words we need to check that if $\gamma: \mathcal{M} \longrightarrow G L(W)$ (with $\gamma=\psi \circ f \circ \iota$ ) then the associated bundle $P_{\mathcal{M}}(W)=P(H)(W)$ is polystable.

Observe that by Def. 3.9 we have a faithful representation $G L(V)$ of $\mathcal{M}$ (from that of $H^{\prime}$ ) such that $P_{\mathcal{M}}(V)$ is polystable of degree zero. Further, we are over a field of characteristic zero and so the $\mathcal{M}$-module $W$ can be realized as a direct summand of the direct sums of some 
$T^{a, b}(V)=V^{\otimes a} \otimes V^{* \otimes b}$ (cf. e.g., [42, p. 86]). (We need the reductivity of $\mathcal{M}$ here, otherwise in general this is only a subquotient and not a direct summand.) Hence the vector bundle $P_{\mathcal{M}}(W)$ is a direct summand of $\bigoplus T^{a, b}\left(P_{\mathcal{M}}(V)\right)$. Since $P_{\mathcal{M}}(V)$ is polystable of degree zero, so is $T^{a, b}\left(P_{\mathcal{M}}(V)\right)$.

By assumption, since $P_{\mathcal{M}}$ is flat the associated vector bundle via any representation is of degree zero. Hence $P_{\mathcal{M}}(W)$ has degree 0 .

Since all bundles have degree zero and $\bigoplus T^{a, b}\left(P_{\mathcal{M}}(V)\right)$ is a direct sum of stable bundles of degree 0 it is easy to check that $P_{\mathcal{M}}(W)$ is also direct sum of stable bundles of degree 0 and hence polystable. q.e.d.

Remark 3.12. The polystability of $P_{\mathcal{M}}(W)$ also follows directly from that of $P_{\mathcal{M}}$ by Remark 2.12. We cannot use Theorem 2.11 as it stands since $\mathcal{M}$ is in general only reductive. But the advantage here is that the bundle $P_{\mathcal{M}}$ comes from a representation of the fundamental group.

After this brief interlude on curves, we now return to the general setting of higher dimensional varieties $X$.

Proposition 3.13. Assume that $\operatorname{dim}(X)=2$. Let $E$ be a rational polystable principal $G$-bundle of degree zero on $U \subset X$. Let $W$ be a $G$-module and $N$ a $G$-subscheme of $W$ of the form $G / H^{\prime}$ where $H^{\prime}=$ $\operatorname{Stab}_{G}(w)$ for some $w \in W$. If $s$ is a section of $E(W)$ such that for $x \in C \subset U$, the image $s(x)=w$ in the fibre of $E(N)$ at $x \in X$, then there exists a big open subset $U^{o} \subset U$ such that the entire image of $U^{o}$ under s lies in $E(N)$. (Here $C \subset U$ is as in (3.2) where the base point $x$ was chosen in a general $C$.) In particular, $E$ has a reduction of structure group to the subgroup $H^{\prime}$ on a big open subset.

Proof. By choice, since $x \in C \subset U$, and $\left.E\right|_{C}$ remains polystable of degree 0, it follows by Prop. 3.8 that $s(C) \subset N$.

Now, again by the Mehta-Ramanathan theorem for $m \gg 0$, there exists an open $\Omega_{m} \subset|m \Theta|$ such that for $t \in \Omega_{m}$, the restriction of $\left.E\right|_{Y_{t}}$ to the smooth projective curve $Y_{t}$ remains polystable of degree 0 . Further, the set $\left\{t \in \Omega_{m} \mid Y_{t} \cap C \neq \emptyset\right\}$ is an open subset of $\Omega_{m}$, which we continue to call $\Omega_{m}$. Since $Y_{t} \cap C \neq \emptyset$, for each $t \in \Omega_{m}$, there is a point $x_{t} \in Y_{t} \cap C$ and hence $s\left(x_{t}\right) \in Y_{t}$. Thus applying Prop. 3.8 to the restriction $\left.E\right|_{Y_{t}}$, we see that $s\left(Y_{t}\right) \subset N$ for all $t \in \Omega_{m}$. Hence, the dense subset $U_{1}=\bigcup Y_{t} \subset U$ is mapped into $N$ i.e., $s\left(U_{1}\right) \subset N$. This implies that the entire image $s(U)$ lies in the closure of the orbit $\bar{N} \subset W$.

Now observe that $N \subset \bar{N}$ is an open subset; therefore, it follows that the set of points $U^{o}=\{u \in U \mid s(u) \in N\}$ is an open subset of $U$.

We claim that $U^{o} \subset U$ is also large. For if not, then the complement of $U_{2}$ in $U$ contains a curve and there are $t \in \Omega_{m}$ such that this curve in the complement meets $Y_{t}$, a contradiction since the entire curve $Y_{t}$ gets mapped into $N$. This proves our claim.

q.e.d. 
Now let $X$ be an smooth projective variety of dimension $d$.

Since $E$ is a rational polystable bundle there is a big $U$ where it is a principal $H$-bundle. We work with the chosen complete intersection as in (3.2) in such a manner that $C \subset U$. With these choices and the point $x \in C \subset U$ we have the following:

Proposition 3.14. Let $E$ be a rational polystable principal $G$-bundle of degree zero on $U \subset X$. Let $W$ be a $G$-module and $N$ a $G$-subscheme of $W$ of the form $G / H^{\prime}$ where $H^{\prime}=S_{\text {Stab }}(w)$ for some $w \in W$. Let $s$ be a section of $E(W)$ (defined on $U$ ) such that the image $s(x)$, of the point $x \in C \subset U$, lies in the fibre of $E(N)$ at $x \in X$. Then there exists $a$ big open subset $U^{o} \subset U$, such that the entire image of $U^{o}$ under $s$ lies in $E(N)$. In particular, $E$ has a reduction of structure group to the subgroup $H^{\prime}$ on a big open subset.

Proof. By choice, since $x \in C \subset U$, and $\left.E\right|_{C}$ remains polystable of degree 0, it follows by Prop. 3.8 that $s(C) \subset E(N)$.

Let us, for the present, denote the divisor $D_{1}$ simply by $D$. By an induction on the dimension $d$, and Prop. 3.13, we see that since $C \subset D^{\prime}=D \cap U$, and since $\left.E\right|_{D^{\prime}}$ is also polystable, there exists a big open subset $D^{o} \subset D^{\prime}$ such that $s\left(D^{o}\right) \subset E(N)$.

Let $Y_{j} \in S$ be any other general divisor. Then, by the property of $S$, we see that $D \cap Y_{j}$ is a smooth divisor of $D$ and $Y_{j}$. Further, by other choices of $Y_{i} \in S$ we see that there exists a smooth projective curve $C_{j} \subset Y_{j} \cap D$.

Since $D^{o} \subset D^{\prime}$ is a big open subset, in fact, it is easy to see that, by going to a smaller open subset of $S$ if need be (and fixing it), we can make sure that $C_{j} \subset D^{o}$ for all $Y_{j} \in S$ (cf. 4.4, [29]).

Again $C_{j} \subset\left(Y_{j} \cap U\right)$ and $\left.E\right|_{\left(Y_{j} \cap U\right)}$ is also a polystable rational bundle. Since $s\left(C_{j}\right) \subset s\left(D^{o}\right) \subset E(N)$, it follows again by induction on dimension that there exists a big open $Y_{j}^{o} \subset Y_{j}$ such that $s\left(Y_{j}^{o}\right) \subset E(N)$ for all $Y_{j} \in S$.

Now as in Prop. 3.13, we see that the set of points $U^{o}=\{u \in$ $\left.U \mid s(u) \in E(N)_{u}\right\}$ is a non-empty open subset of $U$.

We claim that $U^{o} \subset U$ is also large. For if not, then the complement of $U^{o}$ in $U$ contains a divisor $R \subset U$ and there are $Y_{t} \in S$ such that $R \cap Y_{t} \neq \emptyset$. Moreover, by Bertini's theorem one has $\operatorname{dim}\left(R \cap Y_{t}\right)=$ $\operatorname{dim} R-1=\operatorname{dim}\left(Y_{t}\right)-1$. This implies that $\left(R \cap Y_{t}\right) \not \subset\left(Y_{t}-Y_{t}^{o}\right)$, i.e., $R \cap Y_{t}^{o} \neq \emptyset$. That is, there exists $y \in R \cap Y_{t}^{o}$ such that $s(y) \in E(N)_{y}$, which contradicts the fact that $R \subset U-U^{o}$. q.e.d.

Remark 3.15. We work with this $U^{o}$ from now on and since there is no confusion we will call this $U$.

3.5. Completion of proof of Theorem 3.7. We work over the large open $U_{A}$ and all bundles in this proof are are over $U_{A}$. 
By Lemma 3.6, we have an immersion

$$
E_{A}\left(G_{A} / H_{A}^{\prime}\right) \subset E_{A}\left(W_{A}\right) .
$$

The given section $s_{K}^{\prime}$ of $E_{K}\left(G_{K} / H_{K}^{\prime}\right)$ therefore gives a section $u_{K}$ of $E\left(W_{K}\right)$.

Recall also that we have a chosen complete intersection

$$
C \subset \cdots\left(D_{1} \cap D_{2} \cap \cdots \cap D_{c}\right)^{\prime} \cdots \subset D_{1}^{\prime} \subset U
$$

and the point $x \in C$ (cf. Remark 3.2).

Further, $u_{K}(x)$, the restriction of $u_{K}$ to $x \times T^{*}$, extends to give a section $u_{A}(x)$ of $E_{x}\left(W_{A}\right)$ (restriction of $\left.E_{A}\left(W_{A}\right)\right|_{C_{A}}$ to $\left.x \times T\right)$.

Thus by Lemma 2.13, and by the semistability of $E\left(W_{A}\right)_{p}$, on $C$ the section $u_{K}$ extends to give a section $u_{A}$ of $\left.E\left(W_{A}\right)\right|_{C_{A}}$ over $C \times T$.

Again, by Lemma 2.14, we see, by an induction on dimension, that the section $u_{K}$ which is defined on $U_{K}$ extends to section $u_{A}$ of $E_{A}\left(W_{A}\right)$ over the entire open subscheme $U_{A}$.

Now, to prove the Theorem 3.7, we need to make sure that:

(*) The image of this extended section $u_{A}$

$$
\text { actually lands in } E_{A}\left(G_{A} / H_{A}^{\prime}\right) \text {. }
$$

This would then define $s_{A}^{\prime}$.

To prove $(*)$, it suffices to show that $u_{A}(U \times p)$ lies in $E_{A}\left(G_{A} / H_{A}^{\prime}\right)_{p}$ (the restriction of $E_{A}\left(G_{A} / H_{A}^{\prime}\right)$ to $\left.U \times p\right)$.

Observe that $u_{A}(x \times p)$ lies in $E_{A}\left(G_{A} / H_{A}^{\prime}\right)_{p}$ since $u_{A}(x)=s_{A}^{\prime}(x)=$ $w_{A}$.

Observe further that, if $E_{p}$ denotes the principal $G$-bundle on $U$, which is the restriction of the $G_{A}$-bundle $E_{A}$ on $U \times T$ to $U \times p$, then $E_{A}\left(W_{A}\right)_{p}=E_{A}\left(W_{A}\right) \mid(U \times p)$, and we also have

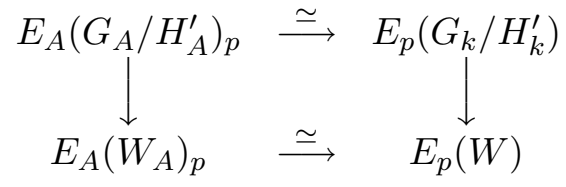

and the vertical maps are inclusions:

$$
E_{A}\left(G_{A} / H_{A}^{\prime}\right)_{p} \hookrightarrow E_{A}\left(W_{A}\right)_{p}, \text { and } E_{p}\left(G_{k} / H_{k}^{\prime}\right) \hookrightarrow E_{p}(W)
$$

where $E_{p}(W)=E_{p} \times{ }_{k}^{\prime} W$ with fibre as the $G$-module $W=W_{A} \otimes k$. Note that $G / H_{k}^{\prime}$ is a $G$-subscheme $Y$ of $W$.

Recall that $E_{p}$ is polystable of degree zero. Then, from the foregoing discussion, the assertion that $u_{A}(U \times p)$ lies in $E_{A}\left(G_{A} / H_{A}^{\prime}\right)$ is a consequence of Proposition 3.14 applied to $E_{p}$ (we might have to throw away a subset of $U$ of codimension $\geq 2$ for this).

Thus we get a section $s_{A}^{\prime}$ of $E_{A}\left(G_{A} / H_{A}^{\prime}\right)$ on $U \times T$, which extends the section $s_{K}^{\prime}$ of $E_{A}\left(G_{A} / H_{A}^{\prime}\right)$ on $U \times T^{*}$. This gives a reduction of structure group of the $G_{A}$-bundle $E_{A}$ on $U \times T$ to the subgroup scheme 
$H_{A}^{\prime}$, and this extends the given bundle $E_{K}$ to the subgroup scheme $H_{A}^{\prime}$.

q.e.d.

\section{Semistable reduction for quasibundles over projective varieties}

The aim of this section is to prove the following theorem.

Theorem 4.1. Let $\mathrm{P}_{K}$ be a family of semistable principal $H$-quasibundles on $X \times \operatorname{Spec} K$, or equivalently, if $H_{K}$ denotes the group scheme $H \times \operatorname{Spec} K$, a semi-stable $H_{K}$-quasibundle $\mathrm{P}_{K}$ on $X_{K}$. Then there exists a finite extension $L / K$, with the integral closure $B$ of $A$ in $L$, such that $\mathrm{P}_{K}$, after base change to $\mathrm{Spec} B$, extends to a semistable $H_{B}$ quasibundle $\mathrm{P}_{B}$ on $X_{B}$.

4.1. Potential good reduction. We begin by observing that we have extended the original rational $H_{K}$-bundle up to isomorphism to a rational $H_{A}^{\prime}$-bundle. To complete the proof of the Theorem 4.1, we need to extend the $H_{K}$-bundle to an $H_{A}$-bundle.

Remark 4.2. We note that in general the group scheme $H_{A}^{\prime}$ obtained above need not be a smooth group scheme over $A$. But in our case, since the characteristic of the base field is zero and since $H_{A}^{\prime}$ is flat, it is also smooth over $A$.

Recall that $H_{A}$ denotes the reductive group scheme $H \times$ Spec $A$ over $A$. We need the following crucial result from [3]. It involves key inputs from Bruhat-Tits theory. For details see Appendix of [4].

Proposition 4.3. There exists a finite extension $L / K$ with the following property: If $B$ is the integral closure of $A$ in $L$, and if $H_{B}^{\prime}$ are the pull-back group schemes, then we have a morphism of B-group schemes

$$
H_{B}^{\prime} \longrightarrow H_{B}
$$

which extends the isomorphism $H_{L}^{\prime} \cong H_{L}$.

We also need the following:

Lemma 4.4. Let $H^{\prime}$ be a non-reductive group and let $\psi: H^{\prime} \longrightarrow H$ be a homomorphism of algebraic groups. Let $P$ be a rational principal $H^{\prime}-$ bundle on the big open subset $U \subset X$ obtained as reduction of structure group of a polystable vector bundle $E$ of degree zero through an inclusion $H^{\prime} \hookrightarrow G L(V)$. Then, by the extension of structure group the rational principal $H$-bundle $P(H)$ is also semistable.

Proof. By the choice of $P$ and by the restriction theorem of Mehta and Ramanathan, we may choose a high degree general smooth complete intersection curve $C$ of type $(m)$, with $C \subset U$ such that $\left.E\right|_{C}$ is polystable of degree zero. Let $a$ denote the product $a_{1} \ldots a_{d-1}$ in the notation of (3.1). 
Thus by Prop. 3.11, the associated $H$-bundle $\left.P(H)\right|_{C}$ is a semistable principal bundle on $C$.

We claim that this implies that $P(H)$ is itself a semistable principal $H$-bundle. For, if $Q \subset H$ is a parabolic subgroup and $\chi$ a dominant character of $Q, P(H)_{Q}$ a $Q$-bundle obtained from a reduction of structure group to $Q$, note that

$$
\operatorname{deg} P(H)_{Q}(\chi) \cdot a^{m}=\operatorname{deg} P(H)_{Q}(\chi)_{\left.\right|_{C}}
$$

where $P(H)_{Q}(\chi)$ denotes the line bundle associated to the character $\chi$. Since $m \gg 0$ it follows by the semistability of $P(H)_{\left.\right|_{C}}$ that $\operatorname{deg} P(H)_{Q}(\chi)_{\left.\right|_{C}}>0$ and hence $\operatorname{deg} P(H)_{Q}(\chi)>0$, i.e., $P(H)$ is semistable.

q.e.d.

Proof of Theorem 4.1. Let the semistable principal $H_{K}$-quasibundle $\mathrm{P}_{K}$ arise out of the faithful representation $H \subset G=G L(V)$. In other words, there is a pair $\left(\mathcal{E}_{K}, \sigma_{K}\right)$ of semistable torsion-free sheaves $\mathcal{E}_{K}$ on $X_{K}$ and section $\sigma_{K}$. By Prop. 2.15, we have a semistable extension $\mathcal{E}_{A}$ of $\mathcal{E}_{K}$ (possibly by going to a finite extension $L / K$ such that $\mathcal{E}_{p}$ is polystable of degree 0$)$. Let $U=U\left(\mathcal{E}_{p}\right)$. Then as we have seen earlier, the entire family $\mathcal{E}_{A}$, when restricted to $U_{A}$, is locally free. Now we are in the setting of Prop. 3.7.

Moreover, the fibre of $E_{B}$ over the closed point is indeed semistable. To see this, observe firstly that it comes as the extension of structure group of $E_{p}^{\prime}$ by the map $\psi_{k}: H_{k}^{\prime} \longrightarrow H_{k}$. Recall (Proposition 3.8) that $E_{p}^{\prime}$ is the semistable $H_{k}^{\prime}$-bundle obtained as the reduction of structure group of the polystable vector bundle $E\left(V_{A}\right)_{p}$ and the semistability of the extended $H_{k}$-bundle follows by Lemma 4.4.

By Proposition 3.7 we have a rational $H_{A}^{\prime}$-bundle $P_{A}^{\prime}$ on $U_{A}$, which extends the $H_{K}$-bundle upto isomorphism. Further, by Proposition 4.3, by going to the extension $L / K$ we have a morphism of $B$-group schemes $\psi_{B}: H_{B}^{\prime} \longrightarrow H_{B}$ which is an isomorphism over $L$. Therefore, one can extend the structure group of the bundle $P_{B}^{\prime}$ to obtain a rational $H_{B^{-}}$ bundle $P_{B}$ which extends the $H_{K}$-bundle $P_{K}$. By using the faithful representation $H \subset G L(V)$, we get a family of vector bundles $P_{B}(V)$ on $U_{B}$.

Note that the rational $H_{K}^{\prime}$-bundle $P_{K}$ is the restriction to $U_{K}$ of the principal $H_{K}$-quasibundle $\left(\mathcal{E}_{K}, \sigma_{K}\right)$.

In other words, we have an isomorphism $P_{L}(V) \simeq \mathcal{E}_{L}$ on $U_{L}$.

Now we apply a result from Langton's paper ([24, Prop. 6]) to conclude that the bundle $P_{B}(V)$ on $U_{B}$ in fact extends to a torsion-free sheaf $\mathcal{E}_{B}$ on $X_{B}$. Further, the reduction section $\sigma_{L}$ (defined on $X_{L}$ has extended to a section $\sigma_{B}$ on $U_{B}$ (given by the principal $H_{B}$-bundle $P_{B}$ ). We can view the reduction datum as follows:

$$
\sigma_{B}:\left(U_{B} \cup X_{L}\right) \longrightarrow\left(S_{\mathcal{E}_{B}}\right) / / H_{B}
$$


(which by the extension property, agrees on the intersection). The complement of $U_{B} \cup X_{L}$ in $X_{B}$ is a subset of points of codimension $\geq 3$, and one can embed the affine scheme $\left(S_{\mathcal{E}_{B}}\right) / / H_{B}$ as a closed subscheme of a vector bundle over $X_{B}$.

This implies that the section $\sigma_{B}$ extends to a section $\sigma_{B}: X_{B} \longrightarrow$ $\left(S_{\mathcal{E}_{B}}\right) / / H_{B}$. In other words, the pair $\left(\mathcal{E}_{K}, \sigma_{K}\right)$ has been semistably extended (up to isomorphism) to a pair $\left(\mathcal{E}_{B}, \sigma_{B}\right)$, i.e., the principal $H_{K^{-}}$ quasibundle $\mathrm{P}_{K}$ has been semistably extended (up to isomorphism) to principal $H_{B}$-quasibundle $\mathrm{P}_{B}$, possibly after going to a finite extension $B / A$. This completes the proof of Theorem 4.1.

q.e.d.

For purposes of applications later we isolate the following easier half of the Mehta-Ramanathan restriction theorem:

Lemma 4.5. Let $\mathrm{P}$ be an $H$-quasibundle. Then $\mathrm{P}$ is $\mu$-polystable (resp. stable) if $\left.\mathrm{P}\right|_{C}$ is so for a general high degree smooth complete intersection curve $C \subset X$.

Proof. The proof is along the lines of the first part of the proof of Prop. 4.4. For more general results along similar lines cf. [7], whose methods work easily enough for rational principal bundles and hence for quasibundles.

\section{Construction of the moduli space of bundles over surfaces}

From now on $X$ will be a smooth projective surface. The aim of this section is to give an algebro-geometric construction of the DonaldsonUhlenbeck compactification for the moduli space of semistable principal $H$-bundles. This in particular also describes geometrically the YangMills compactification of the moduli space of ASD connections on principal bundles with arbitrary structure groups. The method of proof is along the lines of the proof of J. Li (cf. [26]) and the methods in the paper of Le Potier ([25]) (cf. also [21, Chapter 8]).

Remark 5.1. We remark that the salient point in the DonaldsonUhlenbeck compactification which distinguishes it from the MaruyamaGieseker compactification in the usual case of torsion-free sheaves is that, in the boundary of the open subset of $\mu$-stable bundles, various torsion-free sheaves get identified by what may be termed the Uhlenbeck equivalence. It was observed by Donaldson, and used by Le Potier and $\mathrm{Li}$, that the pull-back of a certain determinant line bundle by the morphism defined by "restriction to a general curve" on the moduli detects this collapse of semistable torsion-free sheaves under the new equivalence. It is this observation which leads to the algebro-geometric construction of this compactification. A similar phenomenon is seen in the setting for bundles with arbitrary structure groups. See Prop. 6.14 for the detailed description of the equivalence. 
5.0.1. Double duals for quasibundles. If $\left.\mathrm{P}\right|_{U}$ is a rational $H$-bundle, where $U$ is a big open subset of $X$, then by a theorem of Colliot-Thélène and Sansuc ([10, Theorem 6.7]) there is a principal bundle (unique up to isomorphism) which extends $\left.\mathrm{P}\right|_{U}$. This extension is therefore also associated to each quasibundle $\mathrm{P}$ as well.

Definition 5.2 (Double duals). We call this extended principal bundle the double dual of the quasibundle $\mathrm{P}$ and denote it by $\mathrm{P}^{* *}$. This plays the role of the double dual of torsion free sheaves.

Remark 5.3. If $\mathrm{P}$ is a $\mu$-semistable (resp. polystable, stable) $H-$ quasibundle, then since these definitions involve only big open subsets, the corresponding double dual $\mathrm{P}^{* *}$ is a $\mu$-semistable (resp. polystable, stable) principal $H$-bundle.

5.0.2. On determinant line bundles. Recall that if $\mathcal{F}$ is a flat family of coherent sheaves on $X$ parametrized by a scheme $S$, then $\mathcal{F}$ defines an element $[\mathcal{F}] \in K^{0}(S \times X)$, the Grothendieck group of $S \times X$ generated by locally free sheaves. We may then define the homomorphism from the Grothendieck group of coherent sheaves on $X$ given by:

$$
\lambda_{\mathcal{F}}: K(X) \longrightarrow \operatorname{Pic}(S) .
$$

This has a collection of functorial properties for which we refer to $([\mathbf{2 1}]$ p. 179). For every class $u \in K(X)$ we denote the associated class of the line bundle by $\lambda_{\mathcal{F}}(u)$. Fix a class $c \in K(X)$ with rank $r$ and Chern classes $c_{1}=\mathcal{O}_{X}$ and $c_{2}$, the very ample divisor $\Theta$ on $X$ and a base point $x \in X$. Then there is a natural choice of a class $u_{1}(c) \in K(X)$ defined in terms of these fixed data (cf. p. 184 [21]).

5.0.3. The parameter space for quasibundles. We first briefly recall the construction of the parameter space of $\mu$-semistable torsionfree sheaves over the surface $X$. Let $c \in K(X)$ be as above. Then one knows that the set $\mathcal{S}$ of isomorphism classes of $\mu$-semistable torsion-free sheaves of class $c$ with trivial determinant and fixed Hilbert polynomial $P$ is bounded and hence for a $m \gg 0$ we can realize them as points of a suitable quot scheme. Fix such an $m$. Let $W$ be a complex vector space of dimension $P(m)$.

Let $R^{\mu s s} \subset$ Quot $(W, P)$ be the locally closed subscheme of all quotients $\left[q: W \otimes \mathcal{O}_{X}(-m) \longrightarrow \mathcal{E}\right]$ such that $\mathcal{E}$ is $\mu$-semistable of rank $r$ with trivial determinant and second Chern class $c_{2}$, with Hilbert polynomial $P$ and such that $q$ induces an isomorphism $W \simeq H^{0}(\mathcal{E}(m))$.

There is a natural action of $\mathcal{G}=S L(W)$ on $R^{\mu s s}$ and we have the universal quotient $q_{Q}: \pi_{X}^{*} \mathcal{O}_{X}(-m) \otimes W \longrightarrow \mathcal{E}_{Q}$.

Returning to our setting, let $\rho: H \hookrightarrow G$ be a faithful representation of $H$ in $G=S L(V)$ where $\operatorname{dim}(V)=r$. We shall use the following notation when we wish to stress the fact the quot scheme as parametrizing 
semistable principal quasibundles with structure group $G$ rather than torsion-free sheaves of rank $r$ :

$$
R_{G}:=R^{\mu s s} .
$$

Denote the universal quotient on $X \times R_{G}$ by $q_{R_{G}}: \pi_{X}^{*} \mathcal{O}_{X}(-m) \otimes W \longrightarrow$ $\mathcal{E}_{G}$.

Recall our notation of $\S 2$ and the notion of quasibundles with respect to the representation $\rho$. These are obtained from the affine $X$-scheme $S_{\mathcal{E}}$ together with a generalised reduction datum $\sigma: X \longrightarrow S_{\mathcal{E}} / / H$. The pair $(\mathcal{E}, \sigma)$ is a principal quasibundle with structure group $H$. We remark that this reduction datum $\sigma$ can be equivalently viewed as giving an $\mathcal{O}_{X}$-algebra morphism $\tau:\left(\operatorname{Sym}^{*}(\mathcal{E} \otimes V)\right)^{H} \longrightarrow \mathcal{O}_{X}$. The map $\tau$ which come from a genuine generalised reduction $\sigma$ is not simply the projection onto $\mathcal{O}_{X}$. It is obtained by dualizing the map $\sigma$, where $\left.\sigma\right|_{U}$ is a reduction of structure group of the principal bundle (associated to $\left.\left.\mathcal{E}\right|_{U}\right)$ to $H$ on a large open subset $U=U(\mathcal{E})$.

Let $R_{H}(\rho)$ be the scheme which parametrizes pairs $(q, \tau)$ where $q \in$ $R_{G}$ and $\tau$ is a reduction datum giving a quasibundle with structure group $H$, and let $R_{H}(\rho)^{o}$ be the open subset of pairs $(q, \tau)$ such that $\tau$ defines a principal bundle with structure group $H$.

The existence of total families for principal quasibundles follows by the general theory of Hilbert schemes and is shown in ([37]); in Schmitt's language these will be parametrizing what he terms honest singular bundles. We have been somewhat loose in defining the parameter space but we refer the reader to Schmitt's paper (cf. [37, Section 6.7]) for details.

The scheme $R_{H}(\rho)$ is an $R_{G}$-scheme and the natural map $f_{\rho}: R_{H}(\rho)$ $\longrightarrow R_{G}$ is the one induced by $\rho$. Therefore, since we have already fixed the Chern classes of the torsion free sheaves in $R_{G}$, by the general theory of characteristic classes for principal bundles, the characteristic classes of the principal bundles in the open subscheme $R_{H}(\rho)^{0} \subset R_{H}(\rho)$ will also be canonically fixed. This follows by the basic result of BorelHirzebruch which relates the characteristic classes of principal bundles with the Chern classes of associated vector bundles. For a nice exposition and explicit results cf. [6, Prop. 3.2].

For simplicity we will denote this entire datum by c (we will return to this in 6.2.1).

Remark 5.4. It is immediate that the $\mathcal{G}$-action on $R_{G}$ lifts to an action on $R_{H}(\rho)$.

By the universal property of the scheme $R_{H}(\rho)$ it follows that there exists a tautological family on $X \times R_{H}(\rho)$. Let $\mathcal{P}$ denote this $R_{H}(\rho)$-flat family of $H$-quasibundles on $X$ associated to the faithful representation $\rho$. 
Then by its definition, there exists an $R_{H}(\rho)$-flat family of torsion free sheaves $\mathcal{P}(\rho)=\mathcal{F}$ on $X \times R_{H}(\rho)$. This is given precisely by the family $\left(i d_{X} \times f_{\rho}\right)^{*}\left(\mathcal{E}_{G}\right)$ obtained from the family of semistable torsion free sheaves on $X \times R_{G}$. As remarked above, there is $\mathcal{G}$-action on $R_{H}(\rho)$ such that the family $\mathcal{F}$ carries a linearization with respect to the $\mathcal{G}$-action.

Definition 5.5. Let $\mathcal{P}(\rho)=\mathcal{F}$ be the induced family of torsion free sheaves on $X$ parametrized by $R_{H}(\rho)$. Fixing the class $c \in K(X)$ as seen above, we have a canonical choice of $u_{1}(c) \in K(X)$. We then have the determinant line bundles $\mathcal{L}:=\lambda_{\mathcal{F}}\left(u_{1}(c)\right)$ on $R_{H}(\rho)$ induced by the family $\mathcal{F}$.

Notation. Since we have emphasized the role of the representation $\rho$, we will henceforth denote the total space $R_{H}(\rho)$ simply by $R_{H}$.

Then we have the following:

Lemma 5.6 ([21, Lemma 8.2.4]).

1. If $s \in S$ is a point such that $\left.\mathcal{P}_{s}\right|_{C}$ is $\mu$-semistable, then there exists an integer $n>0$, and $\mathcal{G}$-invariant sections $\bar{\sigma}$ in $\lambda_{F}\left(u_{1}(c)\right)^{n}$, such that $\bar{\sigma}(s) \neq 0$.

2. Let $s_{1}$ and $s_{2}$ be two points in $S$ such that either $\left.\mathcal{P}_{s_{1}}\right|_{C}$ and $\left.\mathcal{P}_{s_{2}}\right|_{C}$ are both semistable but not $S$-equivalent (in the sense of Seshadri) or one of them is semistable and the other is not. Then, in either case, there are $\mathcal{G}$-invariant sections in some tensor power of $\lambda_{F}\left(u_{1}(c)\right)$ that separate $s_{1}$ and $s_{2}$.

Proof. Since the proof follows the general line as given in the text ([21]), we content ourselves by giving the main steps in the argument and refer the reader to $[\mathbf{2 1}]$ for more details.

We need to relate the "determinant line bundle" on $R_{H}$ to its pullback from the corresponding total family $Q_{C}$ of principal $G$-bundles on a high degree curve $C \subset X$.

Let $C$ be a general smooth curve in $X$ of high degree $a \gg 0$. Let $i: C \hookrightarrow X$. For the class $c \in K(X)$, its restriction to $\left.c\right|_{C}:=i^{*}(c)$ in $K(C)$ is completely determined by its rank $r$ since we have assumed that all our sheaves have trivial determinants. Let $P^{\prime}$ be the Hilbert polynomial determined on $C$ by this restriction. Then for a large positive integer $m^{\prime}$ we consider the induced "quot scheme" of quotients $\left[q: W^{\prime} \otimes \mathcal{O}_{C}\left(-m^{\prime}\right) \longrightarrow E\right]$. Let us denote this quot scheme by $Q_{C}$. Here $W^{\prime}$ is a complex vector space of dimension $P^{\prime}\left(m^{\prime}\right)$. Let $Q_{C}^{s s}$ be the quotients which give semistable bundles on $C$ and let $q_{C}$ : $W^{\prime} \otimes \pi_{C}^{*}\left(\mathcal{O}_{C}\left(-m^{\prime}\right)\right) \longrightarrow \mathcal{E}_{C}$ be the universal quotient on $X \times Q_{C}$ with determinant line bundle $\mathcal{L}_{0}^{\prime}$ on $Q_{C}$ obtained from this family. We then have the following properties on separating points in $Q_{C}$ by sections of the determinant line bundle $\mathcal{L}_{0}^{\prime}$ : 
(a) All $S L\left(P^{\prime}\left(m^{\prime}\right)\right)$-semistable points (in the GIT sense) in $Q_{C}$ are precisely the bundles $E$ which are semistable. Further, there exist an integer $\nu$ and an $S L\left(P^{\prime}\left(m^{\prime}\right)\right)$-invariant section $\sigma$ of $\mathcal{L}_{0}^{\prime \nu}$ such that $\sigma([q]) \neq 0$, where $q \in Q_{C}^{s s}$ corresponds to the bundle $E$.

(b) Two points $q_{i}, i=1,2$ in $Q_{C}$ are separated by invariant sections in some tensor power of $\mathcal{L}_{0}^{\prime}$ if and only if either both are semistable but not $S$-equivalent, or one of them is semistable and the other is not.

We now work with $R_{H}$ and the family $\mathcal{F}$ on $X \times R_{H}$. Let us denote the restriction of $\mathcal{F}$ to $C \times R_{H}$ by $\mathcal{F}_{C}$.

Now by increasing $m^{\prime}$ if necessary, we can make sure that:

- The restrictions $\left.\mathcal{F}_{s}\right|_{C}$ are points of $Q_{C}$.

- The sheaf $p_{*} \mathcal{F}_{C}\left(m^{\prime}\right)$ is a locally free $\mathcal{O}_{R_{H}}$-module of rank $P^{\prime}\left(m^{\prime}\right)$, where $p: C \times S \longrightarrow S$ is the projection.

Let $S_{H}$ be the associated projective frame bundle and let $\eta: S_{H} \longrightarrow$ $R_{H}$ be the natural map. It parametrizes a quotient

$$
\mathcal{O}_{S_{H}} \otimes \pi_{C}^{*} \mathcal{O}_{C}\left(-m^{\prime}\right) \otimes W^{\prime} \longrightarrow \eta^{*} \mathcal{F}_{C} \otimes \mathcal{O}_{\eta}(1) \text {. }
$$

This induces a $S L\left(P^{\prime}\left(m^{\prime}\right)\right)$-invariant morphism

$$
\Phi_{\mathcal{F}_{C}}: S_{H} \longrightarrow Q_{C} .
$$

We also have the $\mathcal{G}$-action on $\mathcal{F}$ and via this action we get a $\mathcal{G}$-action on $S_{H}$ which commutes with the $S L\left(P^{\prime}\left(m^{\prime}\right)\right)$-action such that both $\eta: S_{H} \longrightarrow R_{H}$ and $\Phi_{\mathcal{F}_{C}}$ are equivariant for the $\mathcal{G} \times S L\left(P^{\prime}\left(m^{\prime}\right)\right)$-actions.

One then shows by a degree computation that:

$$
\Phi_{\mathcal{F}_{C}}^{*}\left(\mathcal{L}_{0}^{\prime}\right)^{\operatorname{deg} C} \simeq \eta^{*} \mathcal{L}^{a^{2} \operatorname{deg}(X)}
$$

(The canonical det line bundle on $R_{H}$ depends only on the choice of a polarization on $X$ and the relation \# is independent of $C \in|a \Theta|$; cf. [21, pp. 187-188].)

Since $S_{H} \longrightarrow R_{H}$ is a principal $P G L\left(P^{\prime}\left(m^{\prime}\right)\right)$-bundle, a section invariant under $S L\left(P^{\prime}\left(m^{\prime}\right)\right)$ descends to give a $\mathcal{G}$-invariant section. Thus, for any $\nu$ we get a linear map:

$$
s_{\mathcal{F}}: H^{0}\left(Q_{C},\left(\mathcal{L}_{0}^{\prime}\right)^{\nu \operatorname{deg}(C)}\right)^{S L\left(P^{\prime}\left(m^{\prime}\right)\right)} \longrightarrow H^{0}\left(R_{H}, \mathcal{L}^{\nu a^{2} \operatorname{deg}(X)}\right)^{\mathcal{G}} .
$$

To conclude the proof of the lemma we first observe that the semistability of the quasibundle $\mathcal{P}_{s}$ is equivalent to the $\mu$-semistability of the torsion-free sheaf $\mathcal{F}_{s}$ for each $s \in R_{H}$. Hence by using section of powers of the line bundle $\mathcal{L}_{0}^{\prime}$ on $Q_{C}$ and via the map $s_{\mathcal{F}}$ we can indeed separate the restrictions of quasibundles in $R_{H}$ to $C$ as well.

q.e.d.

Remark 5.7. By the Mehta-Ramanathan theorem, if the degree of the curve $C$ is large enough, then the restriction of a $\mu$-semistable torsion-free sheaf on $X$ to the curve $C$ is semistable. But the same 
curve $C$ may not work for all the bundles in $R_{H}$. Hence we need to work with the whole of $Q_{C}$.

We have the following immediate corollary from the first part of Lemma 5.6:

Corollary 5.8. There exists an integer $\nu>0$ such that the line bundle $\mathcal{L}^{\nu}$ on $R_{H}$ is generated by $\mathcal{G}$-invariant global sections i.e., $\mathcal{L}$ is $\mathcal{G}$-semi-ample.

Since $R_{H}$ is a quasi-projective scheme and since $\mathcal{L}$ is $\mathcal{G}$-semi-ample, there exists a finite dimensional vector space $J \subset J_{\nu}:=H^{0}\left(R_{H}, \mathcal{L}^{\nu}\right)^{\mathcal{G}}$ that generates $\mathcal{L}^{\nu}$. Note that there is nothing canonical in the choice of $J$.

Let morphism $\phi_{J}: R_{H} \rightarrow \mathbb{P}(J)$ be the induced $\mathcal{G}$-invariant morphism defined by the sections in $J$. Because of the nonuniqueness of $J$, each choice of subspace of invariant sections gives rise to a different map $\phi_{J^{\prime}}$ to a different projective space $\mathbb{P}\left(J^{\prime}\right)$.

Definition 5.9. We denote by $M_{J}$ the schematic image $\phi_{J}\left(R_{H}\right)$ with the canonical reduced scheme structure.

Remark 5.10. By the following result which may be titled $\mathcal{G}$-properness, the variety $M_{J}$ is proper and hence because of its quasi-projectivity it is a projective variety. We note that we use the term variety in a more general sense of an reduced algebraic scheme of finite type which need not be irreducible. So in what follows we will be working with the $\mathbb{C}$-valued points of $M_{J}$.

Proposition 5.11. If $T$ is a separated scheme of finite type over $k$, and if $\phi: R_{H} \longrightarrow T$ is an $\mathcal{G}$ invariant morphism, then image of $\phi$ is proper over $k$.

Proof. This is an immediate consequence of Theorem 4.1 and [21, Prop. 8.2.6].

Let $J_{\nu}$ denote the vector space $H^{0}\left(R_{H}, \mathcal{L}^{\nu}\right)^{\mathcal{G}}, \nu \in \mathbb{Z}^{+}$; and Let $J \subset J_{\nu}$ be a finite dimensional vector space which generates $\mathcal{L}^{\nu}$.

For any $d \geq 1$, let $J^{d}$ be the image of the canonical multiplication map $f_{d}: J \otimes, \cdots, \otimes J(d-$ fold $) \rightarrow J_{d \nu}$; in particular, $J^{1}=J$.

Let $J^{\prime}$ be any finite dimensional vector subspace of $J_{d \nu}$ containing $J^{d}$. Then clearly the line bundle $\mathcal{L}^{d \nu}$ is also globally generated by $\mathcal{G}$ invariant sections coming from the subspace $J^{\prime}$ and this is so for any $d \geq 0$.

So we have inclusions $J \hookrightarrow J^{d} \hookrightarrow J^{\prime}$, and hence a commutative diagram

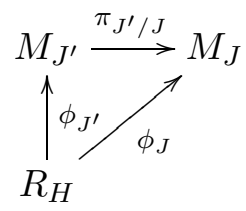


Since $M_{J}$ and $M_{J}^{\prime}$ are both projective, the map $\pi_{J^{\prime} / J}$ is a finite map. So if we fix a $J$ as above, we get an inverse system (indexed by the $d \geq 1)$ of projective varieties $\left(M_{J^{\prime}}, \pi_{J^{\prime} / J}\right)$ and dominated by the finite type scheme $R_{H}$.

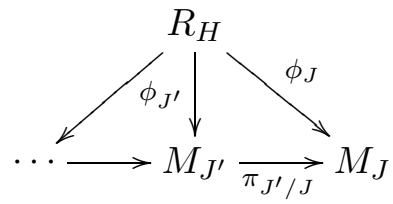

Hence the inverse limit of the system $\left(M_{J^{\prime}}, \phi_{*}\right)$ is in fact one of the $M_{J^{\prime}}$ 's where $J^{\prime}$ is a finite dimensional subspace of $H^{0}\left(R_{H}, \mathcal{L}^{n}\right)^{\mathcal{G}}$ which generates $\mathcal{L}^{n}$.

Definition 5.12. We denote this inverse limit variety by $M_{H}(\rho)$ and let $\pi: R_{H} \rightarrow M_{H}(\rho)$ be the canonical morphism induced by the invariant sections coming from the subspace $J_{0}$ associated to the inverse limit.

We summarize the above discussion in the following theorem:

Theorem 5.13. The reduced projective scheme $M_{H}(\rho)$ parametrizes equivalence classes of $\mu$-semistable quasibundles with structure group $H$. There is a natural morphism $q: R_{H} \longrightarrow M_{H}(\rho)$. Furthermore, the representation $\rho: H \longrightarrow G=S L(V)$ induces a natural morphism $\bar{f}_{\rho}: M_{H}(\rho) \longrightarrow M_{G}$, where $M_{G}:=M_{S L(V)}$ is the moduli space of the $\mu$-semistable torsion free sheaves with trivial determinant and fixed $c_{2}$.

Proof. The existence of the morphism $\bar{f}_{\rho}: M_{H}(\rho) \longrightarrow M_{G}$ follows by the naturality of the moduli space $M_{G}$ by virtue of the existence of the family of semistable torsion free sheaves $\mathcal{F}$ on $R_{H}(\rho)$ q.e.d.

Remark 5.14. The strategy is somewhat similar to that of $[\mathbf{3}]$, but unlike in [3], there are no GIT quotients involved here.

Remark 5.15. Note that this is not a categorical quotient since $\mathcal{L}$ is not ample and is only semi-ample (Cor. 5.8), i.e., some power of $\mathcal{L}$ is generated by sections.

5.1. Towards the description of the moduli space. In order to get a better understanding of the geometry of the moduli space $M_{H}(\rho)$ we need the following lemmas:

Lemma 5.16. Let $P_{1}$ and $P_{2}$ be two polystable principal $H$-bundles on $X$. Let $a \gg 0$ and $C \in|a \Theta|$ be a general smooth curve. Then $P_{1} \simeq P_{2}$ if and only if $\left.\left.P_{1}\right|_{C} \simeq P_{2}\right|_{C}$.

Proof. Consider the principal $H \times H$-bundle $P_{1} \times_{X} P_{2}$. Let us denote this bundle by $E$. 
By ([39], p. 19), giving an isomorphism between $P_{1}$ and $P_{2}$ is equivalent to giving a reduction of structure group of $E$ to the diagonal embedding $\Delta \subset(H \times H)$.

By the Chevalley semi-invariant theorem, we can embed $(H \times H) / \Delta \subset$ $W$ in a $H \times H$-module $W$ as a closed orbit.

Thus a section of $E\left(\frac{H \times H}{\Delta}\right)$ is a section of the vector bundle $E(W)$ which lies in $E\left(\frac{H \times H}{\Delta}\right)$.

Claim. The principal bundle $E$ is also polystable of degree 0 . Let us assume this claim and complete the proof.

By the usual Enriques-Severi lemma for a general high degree curve $C$, the restriction map:

$$
H^{0}(X, E(W)) \longrightarrow H^{0}\left(C,\left.E(W)\right|_{C}\right)
$$

is surjective. Further, by assumption, for high degree curves we know that $\left.\left.P_{1}\right|_{C} \simeq P_{2}\right|_{C}$. Hence, by our discussion above we have a reduction of structure group of $\left.E\right|_{C}$ to $\Delta$. i.e., a section:

$$
\sigma_{C}:\left.\left.C \longrightarrow E\left(\frac{H \times H}{\Delta}\right)\right|_{C} \hookrightarrow E(W)\right|_{C}
$$

By (\&) above, there exists a lift of $\sigma_{C}$ to a section $\sigma: X \longrightarrow E(W)$. We need to show that the image of $\sigma$ lies in $E\left(\frac{H \times H}{\Delta}\right)$.

One knows that $E$ is polystable of degree 0 , by the Claim above. Thus we are in the setting of Prop. 3.13. Thus we can get a big open subset $U \subset X$ such that the image of the section $\sigma(U) \subset E\left(\frac{H \times H}{\Delta}\right)$.

Now since $\frac{H \times H}{\Delta} \subset W$ is a closed embedding, it follows that the entire image $\sigma(X) \subset E\left(\frac{H \times H}{\Delta}\right)$. This gives the required reduction of structure group to $\Delta$. To complete the proof we need to prove the claim.

To see this we again use the easier half of the Mehta-Ramanathan restriction theorem (i.e., Lemma 4.5). Thus to show that $E$ is polystable of degree 0 we need to show that for a general high degree curve $C$, the restriction $\left.E\right|_{C}$ is polystable of degree zero.

Since $\left.P_{1}\right|_{C}$ and $\left.P_{2}\right|_{C}$ are polystable of degree 0 (by the restriction theorem again), it follows by Ramanathan's theorem ([34]) (the Narasimhan-Seshadri theorem for principal bundles on curves) that there exist representations $\rho_{i}: \pi_{1}(C) \longrightarrow K i=1,2, K$ a maximal compact subgroup of $H$, such that $\left.P_{i}\right|_{C}$ is the $H$-bundle associated to $\rho_{i}$, for $i=1,2$.

It is easy to see that the bundle $\left.E\right|_{C}$ is the bundle associated to the representation $\rho_{1} \times \rho_{2}([\mathbf{3 4}]$ p. 146) and the polystability follows by Ramanathan's theorem again. This completes the proof of the lemma.

q.e.d.

5.1.1. Associated graded of a semistable quasibundle. Let $\mathrm{P} \longrightarrow$ $X$ be an $H$-quasibundle. Let $\rho: H \hookrightarrow G L(V)$ be the accompanying faithful representation and $\mathcal{E}=\mathrm{P}(\rho)$ the associated torsion-free sheaf. 
The sheaf $\mathcal{E}$ possesses a Jordan-Holder filtration $J^{\bullet}$ by saturated subsheaves and we may take the associated graded sheaf $\operatorname{gr}_{\mathrm{J}}(\mathcal{E})$. For two different filtrations $J_{i}^{\bullet}$ we have an isomorphism of vector bundles $\left(g r_{J_{1}^{\bullet}}(\mathcal{E})\right)^{* *} \simeq\left(g r_{J_{2}^{\bullet}}(\mathcal{E})\right)^{* *}$ By an abuse of notation we write $g r^{\mu}(\mathcal{E})$ to denote an associated graded torsion-free sheaf.

Let $\mathbb{A}=\mathbb{A}_{\mathbb{C}}^{1}$. Recall that there exists a family $\left\{\mathcal{E}_{t}\right\}_{t \in \mathbb{A}}$ of torsion-free sheaves such that $\mathcal{E}_{t} \simeq \mathcal{E}$ for $t \neq 0$ and $\mathcal{E}_{0} \simeq g^{\mu}(\mathcal{E})$.

By the Semistable reduction Theorem 4.1 (and its proof) we have an $H_{A}$-quasibundle (where $\operatorname{Spec} A=\mathbb{A}_{1} \subset \mathbb{A}$ an open subset which contains 0). Denote this $H_{A}$-quasibundle by $\left\{\mathrm{P}_{t}\right\}_{t \in \mathbb{A}_{1}}$. Further, this family has the property that $\mathrm{P}_{t} \simeq \mathrm{P}$ for $t \neq 0$ and there is a big open set $U$ and a rational $H^{\prime}$-bundle $\mathrm{P}_{0}^{\prime}$ on $U$, such that $\left.\mathrm{P}_{0}\right|_{U}$ is obtained from $\mathrm{P}_{0}^{\prime}$ by an extension of structure group via $\psi: H^{\prime} \longrightarrow H$ (recall that $H^{\prime}$ could be a non-reductive group; cf. Prop. 4.3 and Lemma 4.4).

Also from the proof (Theorem 3.7) one knows that $\left.\mathrm{P}_{0}^{\prime}(\rho) \simeq \mathcal{E}_{0}\right|_{U}$ which is polystable.

Claim. The quasibundle $\mathrm{P}_{0}$ is also polystable.

By Lemma 4.5 it is enough to show that $\left.\mathrm{P}_{0}\right|_{C}$ is polystable, where $C$ is a high degree curve contained in the big open subset $U$, where $\mathrm{P}_{0}$ is a principal bundle. Further, $\left.\mathrm{P}_{0}^{\prime}\right|_{C}$ is a bundle which is flat, i.e., it comes from a representation $\chi_{1}: \pi_{1}(C) \longrightarrow H^{\prime}$ (see Prop. 3.8). Moreover, $\left.\left.\mathrm{P}_{0}^{\prime}(\rho)\right|_{C} \simeq \mathcal{E}_{0}\right|_{C}$ which is polystable by the Mehta-Ramanathan theorem, since $\left.\mathcal{E}_{0}\right|_{U}$ is polystable. In other words, under our definition of polystability of principal bundles with non-reductive structure groups (i.e., Def. 3.9), the bundle $\left.\mathrm{P}_{0}^{\prime}\right|_{C}$ is polystable.

Again $\left.\mathrm{P}_{0}\right|_{C}$ comes from $\left.\mathrm{P}_{0}^{\prime}\right|_{C}$ by extension of structure group $f$ : $H^{\prime} \longrightarrow H$. Therefore, since $\left.\mathrm{P}_{0}^{\prime}\right|_{C}$ is polystable by Prop. 3.11 , it follows that $\left.\mathrm{P}_{0}\right|_{C}$ is polystable. This proves the claim.

Definition 5.17. Let $\mathrm{P}$ be a semistable $H$-quasibundle. If there exists a family $\left\{\mathrm{P}_{t}\right\}_{t \in \operatorname{Spec} A}$, with $A$ a complete discrete valuation ring, such that $\mathrm{P}_{t} \simeq \mathrm{P}$, for $t \neq 0$ and $\mathrm{P}_{0}$ polystable, then we call $\mathrm{P}_{0}$ an associated graded quasibundle of $P$.

This is uniquely defined up to double duals in the following sense:

Lemma 5.18. Let $\mathrm{P}$ be an $H$-quasibundle. Then if $\mathrm{P}_{s_{0}}$ and $\mathrm{P}_{t_{0}}$ are two choices of polystable limits (as above), then $\mathrm{P}_{s_{0}}^{* *} \simeq \mathrm{P}_{t_{0}}^{* *}$.

Proof. Let $\mathrm{P}_{S}$ and $\mathrm{P}_{T}$ be two families of quasibundles such that at the closed points $s_{0} \in S$ and $t_{0} \in T$ the quasibundles $\mathrm{P}_{s_{0}}$ and $\mathrm{P}_{t_{0}}$ are polystable and the generic fibres in either family are isomorphic to $\mathrm{P}$.

Consider the open subset $U \subset X$ where both $\mathrm{P}_{s_{0}}$ and $\mathrm{P}_{t_{0}}$ are locally free. Then since $S$ and $T$ are spectra of discrete valuation rings, the families $\mathrm{P}_{S}$ and $\mathrm{P}_{T}$ are locally free on $U \times S$ and $U \times T$ respectively. Using the Mehta-Ramanathan theorem, choose a general high degree curve $C \subset U$ so that the restrictions $\left.\mathrm{P}_{s_{0}}\right|_{C}$ and $\left.\mathrm{P}_{t_{0}}\right|_{C}$ are polystable. 
Since $C \subset U$ it follows that both $\left.\mathrm{P}_{s_{0}}\right|_{C}$ and $\left.\mathrm{P}_{t_{0}}\right|_{C}$ are polystable limits of $\left.\mathrm{P}\right|_{C}$ (which is also semistable by openness of semistability). But the associated graded of a semistable principal bundle on a smooth projective curve is uniquely defined (cf. [35]). Hence,

$$
\left.\left.\mathrm{P}_{s_{0}}\right|_{C} \simeq \mathrm{P}_{t_{0}}\right|_{C} \text {. }
$$

Now we apply Lemma 5.16 to the double dual principal bundles on $X$ and we are done.

Remark 5.19. If $\mathrm{P}(\rho)=\mathcal{E}$, then for each choice of associated $H$ quasibundle $\operatorname{gr}^{\mu}(\mathrm{P})=\mathrm{P}_{0}$ defined above, we see that $\mathrm{P}_{0}^{* *}(\rho) \simeq \mathcal{E}_{0}^{* *}$.

\section{The geometry of the moduli of $H$-bundles}

From this section onwards $H$ is a simple algebraic group. In this section we study the points of the moduli space $\overline{M_{H}^{0}}$ intrinsically as well as in relationship with its image points in the moduli space $M_{G}$.

6.1. Cycles associated to quasibundles in $\overline{M_{H}^{0}}$. Let $\mathrm{P}$ be a semistable $H$-quasibundle in $\overline{M_{H}^{0}}$. Therefore there exists a family $\left\{\mathrm{P}_{t}\right\}_{t \in \operatorname{Spec} A}$, with $A$ a complete discrete valuation ring, such that $\mathrm{P}_{t}$ is a semistable principal $H$-bundle, for $t \neq 0$ and $\mathrm{P}_{0} \simeq \mathrm{P}$.

Our aim is to associate a cycle $Z_{\mathrm{P}} \in S^{l}(X)$ of degree $l$ to the quasibundle $\mathrm{P}$ in an intrinsic manner. By this we mean that the pair $\left(\mathrm{P}^{* *}, Z_{\mathrm{P}}\right)$ in the compactified moduli space is independent of the quasibundle (and hence the representation $\rho$ as well). Towards this we work with the chosen representation $\rho$ used in defining the quasibundle $\mathrm{P}$.

6.1.1. The Dynkin index of the representation $\rho$. We recall the notion of Dynkin index $([\mathbf{1 4}],[\mathbf{2 2}],[\mathbf{2}])$ and some basic results from these sources which will play a key role in what follows.

Definition 6.1. Let $\theta$ be a the highest root in $\mathfrak{H}$ the Lie algebra of $H$ and $\mathfrak{s l}(\theta)$ the 3 -dimensional sub-algebra of $\mathfrak{H}$ associated to it. Decompose the $H$-module $V$ into $\mathfrak{s t}(\theta)$-modules as $\oplus V_{i}=V$. Let $\operatorname{dim}\left(V_{i}\right)=m_{i}$. Then we can define the Dynkin index $m_{\rho}$ of the $H_{-}$ module $V$ as follows:

$$
m_{\rho}=\sum_{i}\left(\begin{array}{c}
m_{i}+1 \\
3
\end{array}\right) .
$$

Remark 6.2. The Dynkin index of a simple subgroup $H$ of a simple Lie group $G$ is usually defined as the ratio of the invariant inner product on Lie $(H)$ to the invariant inner product of Lie $(G)$ where the inner products are normalized to make the length of the highest root $2([\mathbf{2}, \mathrm{p}$. $455])$.

\section{Example 6.3.}

(a) Let $\rho_{m}: S L(2) \longrightarrow S L(V)$ be the standard irreducible representation $V=S^{m}(W)$ where $\operatorname{dim}(W)=2$. The $m_{\rho_{m}}=\left(\begin{array}{c}m+2 \\ 3\end{array}\right)$. 
(b) Let $\rho: S L(2) \longrightarrow S L(V)$ be the inclusion obtained by identifying $S L(2)$ with the 3-dimensional group given by the highest root $\theta$ in $\mathfrak{s l}(V)$. Then $m_{\rho}=1$.

(c) If $\rho$ is the adjoint representation of $H$ then the index is $2 h$, where $h$ is the dual Coxeter number of $H$.

The following is the list of index 1 subgroups given by Dynkin $([\mathbf{1 4}])$. We reproduce the list from [2]:

Table 1.

\begin{tabular}{|c|}
$S(U(n) \times U(1)) \subset S U(n+1)$ \\
$\operatorname{Spin}(n) \subset \operatorname{Spin}(n+1)$ \\
$S p(n) \times S p(1) \subset S p(n+1)$ \\
$S U(3) \subset G_{2}$ \\
$\operatorname{Spin}(9) \subset F_{4}$ \\
$F_{4} \subset E_{6}$ \\
$E_{6} \times U(1) \subset E_{7}$ \\
$E_{7} \times S U(2) \subset E_{8}$
\end{tabular}

We now quote from [22] (see also [2, p. 455]);

Proposition 6.4. Let $\rho_{*}: \pi_{3}(H) \longrightarrow \pi_{3}(S L(V))$. Then it is given by "multiplication" by $m_{\rho}$.

Remark 6.5. This in particular proves that any index 1 inclusion $S U(2) \subset S U(n)$ preserves the second Chern class of a principal $S U(2)-$ bundle when we extend structure group to $S U(n)$ since $\pi_{3}(G) \simeq \mathbb{Z}$ classifies principal $G$-bundles on a real ${ }^{4-\text {-sphere }} S^{4}$ and the second Chern class of the bundle classifies it. This also implies that any $S U(n)$-bundle $E$ on $S^{4}$ is obtained as the extension of structure group from $S U(2)$. In particular, the rank $n$ vector bundle underlying $E$ (topologically) splits as a direct sum of a rank 2 bundle and direct sum of $n-2$ trivial line bundles. Thus if the vector bundle underlying $E$ has a nontrivial section $s$ then the section comes from a section of the rank 2 subbundle. Hence the "zero-scheme" $Z(s)$ is represented by $c_{2}(E)$.

For a point $x \in X$, let $B_{\epsilon}(x)$ be an analytic ball of radius $\epsilon$ around $x$. Let $E$ be a vector bundle on $X$ of rank $r$ and let $E_{\left.\right|_{B_{\epsilon}(x)}}$ be its restriction to $B_{\epsilon}(x)$. Suppose further that it is trivial on the boundary $\partial B_{\epsilon}(x)$. Let us denote by $E_{\left.\right|_{B_{\epsilon}(x)}} / E_{\left.\right|_{\partial B_{\epsilon}(x)}}$ the complex vector bundle on the real four sphere $S^{4} \simeq B_{\epsilon}(x) / \partial B_{\epsilon}(x)$ obtained by identifying $E_{\mid \partial B_{\epsilon}(x)}$ to $\mathbb{C}^{\oplus r}$ using the trivialization.

We then have the following result from [26] and [30] extended for rank $r$ bundles. Li and Morgan show it for the rank 2 case but the proofs generalize to the higher rank case. 
Proposition 6.6. Let $\mathcal{E}_{\mathbb{A}}$ be a family of (analytic) torsion-free sheaves of rank $r$ over $B \times \mathbb{A}$ flat over $\mathbb{A}$, where $B$ is a closed ball in the surface $X$ and $\mathbb{A}$ is the affine line. Furthermore let $\mathcal{E}_{\mathbb{A}}$ be locally free on $B \times \mathbb{A}-0$ and $\mathcal{E}_{0}$ be torsion-free with singularity at the origin $0 \in B$. Let $B_{\epsilon, u} \subset B \times\{u\}$ be the 2 -dimensional $\epsilon$-ball centered at $(0, u)$ and let $\mathcal{E}_{u}$ be the restriction of $\mathcal{E}$ to $B_{\epsilon, u}$. Then the smooth trivialization of $\mathcal{E}_{\left.0\right|_{\partial B_{\epsilon, u}}}$ which is induced from a trivialization of $\left(\mathcal{E}_{\left.0\right|_{B_{\epsilon, u}}}\right)^{* *}$ induces a family of trivializations $\beta_{u}: \mathbb{C}^{\oplus r} \times \partial B_{\epsilon, u} \simeq \mathcal{E}_{\left.u\right|_{\partial B \epsilon, u}}$ which depends smoothly on $u$ with $u \in \mathbb{A}$ being $\epsilon$-small. Furthermore, we also have on $S^{4} \simeq B_{\epsilon, u} / \partial B_{\epsilon, u}$ the following:

$$
c_{2}\left(\mathcal{E}_{\left.u\right|_{B_{\epsilon, u}}} / \mathcal{E}_{\left.u\right|_{\partial B_{\epsilon, u}}}\right)=\operatorname{length}\left(\mathcal{E}_{0}^{* *} / \mathcal{E}_{0}\right)_{0} .
$$

Proof. The only new ingredients needed to generalize Li's arguments are:

- Extension of [26, Prop. 6.4] to higher ranks, which has been done by Gieseker and $\mathrm{Li}[\mathbf{1 8}]$.

- When the length $l=1$ then the length $l$ can be realised as the second Chern class of the rank $r$ bundle on $S^{4}$. This is done by $\mathrm{Li}$ in the rank 2 case by getting a section whose zero-scheme has length 1 and is represented by the $c_{2}$. Since we are on $S^{4}$ this argument goes through for the higher rank case as well, as we have seen in Remark 6.5.

q.e.d.

Let $\mathrm{P}_{t_{0}} \in \overline{M_{H}^{0}}$ be a polystable $H$-quasibundle and $\mathrm{P}_{T}$ be a family of semistable principal $H$-bundles with $\mathrm{P}_{t_{0}}$ as limit. Let $\mathcal{E}_{T}=\mathrm{P}_{T}(\rho)$. Let $Z\left(\mathcal{E}_{t_{0}}\right)$ be the cycle associated to the torsion-free sheaf $\mathcal{E}_{t_{0}}$. Recall that $Z\left(\mathcal{E}_{t_{0}}\right) \in S^{l}(X)$ where $l=c_{2}\left(\mathcal{E}_{t_{0}}^{* *}\right)-c_{2}\left(\mathcal{E}_{t_{0}}\right)$. Further the cycle is given by:

$$
Z\left(\mathcal{E}_{t_{0}}\right):=\sum_{x \in X} \operatorname{length}\left(\mathcal{E}_{t_{0}}^{* *} / \mathcal{E}_{t_{0}}\right)_{x} \cdot x .
$$

Theorem 6.7. Let the notations be as above. Let $x \in \operatorname{Supp}\left(Z\left(\mathcal{E}_{t_{0}}\right)\right)$. Then the number length $\left(\mathcal{E}_{t_{0}}^{* *} / \mathcal{E}_{t_{0}}\right)_{x}$ is a multiple of the Dynkin index $m_{\rho}$. In particular, the total degree $l$ of the cycle $Z\left(\mathcal{E}_{t_{0}}\right)$ is also a multiple of $m_{\rho}$.

Proof. Fix a point $x \in \operatorname{Supp}\left(Z\left(\mathcal{E}_{t_{0}}\right)\right)$ and choose a ball $B$ around it and identify $x$ with $0 \in B$. Let $u \in \mathbb{A}$ be the point in a small disk in $\mathbb{A}$ corresponding to $t_{0} \in T$.

By restricting $\mathcal{E}_{T}$ to $B \times \mathbb{A}$, in the notation of Prop. 6.6, we have a family $\mathcal{E}_{\mathbb{A}}$ satisfying the properties given there. Furthermore, we also have the extra datum that the bundles $\mathcal{E}_{t}$ for $t \neq t_{0}$ have reduction of structure group to principal $H$-bundles $\mathrm{P}_{t} \forall t \neq t_{0}$. 
Following the procedure in Proposition 6.6 we get a vector bundle $\mathcal{E}_{\left.u\right|_{B_{\epsilon}, u}} / \mathcal{E}_{\left.u\right|_{\partial B_{\epsilon, u}}}$ such that

$$
c_{2}\left(\mathcal{E}_{\left.u\right|_{B_{\epsilon, u}}} / \mathcal{E}_{\left.u\right|_{\partial B_{\epsilon, u}}}\right)=\operatorname{length}\left(\mathcal{E}_{0}^{* *} / \mathcal{E}_{0}\right)_{0} .
$$

We now observe easily that the vector bundle $\mathcal{E}_{\left.u\right|_{B_{\epsilon, u}}} / \mathcal{E}_{\left.u\right|_{\partial B_{\epsilon, u}}}$ on $S^{4}$ also has a reduction of structure group to $H$. Thus we have a principal $H$-bundle $P_{\epsilon}$ on $S^{4}$ whose extension of structure group by $\rho: H \hookrightarrow$ $S L(V)$ is $\mathcal{E}_{\left.u\right|_{B_{\epsilon, u}}} / \mathcal{E}_{\left.u\right|_{\partial B_{\epsilon, u}}}$.

By Prop. 6.4 it follows that the second Chern class of $\mathcal{E}_{\left.u\right|_{B_{\epsilon, u}}} / \mathcal{E}_{\left.u\right|_{\partial B_{\epsilon, u}}}$ is a multiple of the Dynkin index $m_{\rho}$. Hence length $\left(\mathcal{E}_{0}^{* *} / \mathcal{E}_{0}\right)_{0}$ is a multiple of $m_{\rho}$. This proves the theorem.

q.e.d.

Definition 6.8. Let $\mathrm{P}_{t_{0}} \in \overline{M_{H}^{0}}$. Then we define the cycle $Z\left(\mathrm{P}_{t_{0}}\right)$ as:

$$
Z\left(\mathrm{P}_{t_{0}}\right):=\sum_{x \in X} \frac{\operatorname{length}\left(\mathcal{E}_{t_{0}}^{* *} / \mathcal{E}_{t_{0}}\right)_{x}}{m_{\rho}} \cdot x .
$$

6.2. Points of the moduli. Let $\mathrm{P}$ be an $H$-quasibundle and let $\mathrm{P}(\rho)=$ $\mathcal{E}$. Further, let $\mathcal{E}_{0}$ be an associated graded sheaf of $\mathcal{E}$ and let us denote the double dual $\mathcal{E}_{0}{ }^{* *}$ by $\mathbb{E}$.

Let $Q(\mathbb{E}, l)$ be the quot scheme of torsion quotients of $\mathbb{E}$ of length $l$. By the choice of our $\mathbb{E}$, the torsion-free sheaves $F \in Q(\mathbb{E}, l)$ all have reduction of structure groups to quasibundles.

Notation. Let the induced total family quasibundles coming from $Q(\mathbb{E}, l)$ be denoted by $Q_{H}(\mathbb{E}, l)$. We thus have the following diagram:

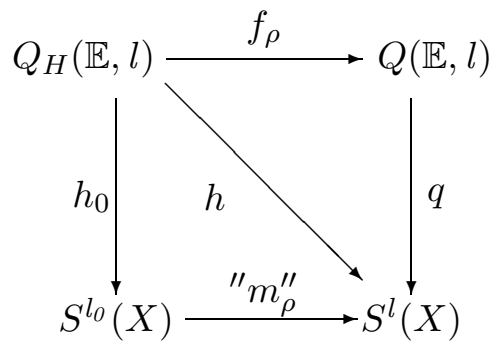

We note the following:

(a) The degree $l_{0}$ is therefore $l_{0}=\frac{l}{m_{\rho}}$.

(b) The vertical map $h_{0}: Q_{H}(\mathbb{E}, l) \longrightarrow S^{l_{0}}(X)$ is the one which associates to each $\mathrm{P}$ the cycle $Z(\mathrm{P})$ defined above.

(c) The map " $m_{\rho}^{\prime \prime}: S^{l_{0}}(X) \longrightarrow S^{l}(X)$ is the map induced by multiplication by the Dynkin index $m_{\rho}$ which maps the cycle $Z(\mathrm{P})$ to $m_{\rho} \cdot Z(\mathrm{P})=Z\left(\mathcal{E}_{0}\right)$. Observe that this map is an injection.

Remark 6.9 (Action of Aut $(\mathbb{E})$ on the fibre of $f_{\rho}$ ). Let $\alpha \in \operatorname{Aut}(\mathbb{E})$. Let $\mathcal{E}$ be a $\mu$-polystable torsion-free sheaf with $\mathbb{E}=g r^{\mu}(\mathcal{E})^{* *}$. Let $\mathrm{P}$ be a quasibundle obtained from a reduction datum $\sigma: X \longrightarrow S_{\mathcal{E}} / / H$. 
We first observe that since $\mathbb{E}$ is a locally free sheaf on $X$, by Hartogs theorem we see that the natural restriction map $\operatorname{res}_{U}:$ Aut $(\mathbb{E}) \longrightarrow$ Aut $\left(\mathbb{E}_{U}\right)$ is an isomorphism. (This holds when $\mathbb{E}$ is reflexive, cf. for example, Cor. 1.11.1 [28]).

By restricting $\alpha$ to $U$ a big open set, we have an action of $\alpha$ on $\sigma_{U}$ (restriction of $\sigma$ to $U$ ). Let $\alpha \cdot \sigma_{U}=\sigma_{U}^{\prime}$. Then by definition $\sigma_{U}^{\prime}: U \longrightarrow$ $S_{\mathcal{E}} / / H$ which extends uniquely to a new datum $\sigma^{\prime}: X \longrightarrow S_{\mathcal{E}} / / H$. We define:

$$
\alpha \cdot \sigma=\sigma^{\prime} .
$$

It is clear that if $\mathrm{P}^{\prime}$ is the quasibundle obtained from $\sigma^{\prime}$, then $\mathrm{P}^{\prime}(\rho)=\mathcal{E}$ and $\mathrm{P}^{* *} \simeq \mathrm{P}^{* * *}$.

We then have the following:

Proposition 6.10. Let $\mathrm{P}$ and $\mathrm{Q}$ be two $\mu$-polystable $H$-quasibundles in $Q_{H}(\mathbb{E}, l)$ lying in the same fibre of $f_{\rho}$. If $\mathrm{P}^{* *} \simeq \mathrm{Q}^{* *}$ then $\mathrm{P}$ and $\mathrm{Q}$ lie in the same connected component of the fibre of the map $h: Q_{H}(\mathbb{E}, l) \longrightarrow$ $S^{l}(X)$ (or equivalently of the map $h_{0}: Q_{H}(\mathbb{E}, l) \longrightarrow S^{l_{0}}(X)$ ).

Proof. We first show that if $\mathrm{P}$ and $\mathrm{Q}$ are two polystable quasibundles in a fibre of the map $f_{\rho}: Q_{H}(\mathbb{E}, l) \longrightarrow Q(\mathbb{E}, l)$ and such that $\mathrm{P}^{* *} \simeq \mathrm{Q}^{* *}$ then $\mathrm{P}$ and $\mathrm{Q}$ lie in the same orbit of the action $A u t(\mathbb{E})$.

Note firstly that the polystability of $P$ and $Q$ as well as the property that they lie in the same fibre of $f_{\rho}$ forces that the associated torsion-free sheaves in $Q(\mathbb{E}, l)$ are $\mu$-polystable. Hence, both $\mathrm{P}$ and $\mathrm{Q}$ are reductions of structure groups of the "same" polystable torsion-free sheaf $\mathcal{E}_{0}$. One also sees that $\mathcal{E}_{0}^{* *} \simeq \mathbb{E}$. In other words, P, Q $\in f_{\rho}^{-1}\left(\mathcal{E}_{0}\right)$.

Consider the restriction to a big open subset $U=U\left(\mathcal{E}_{0}\right)$. By definition, both $\mathrm{P}_{U}$ and $\mathrm{Q}_{U}$ are reductions of structure group of $\mathcal{E}_{U} \simeq \mathbb{E}_{U}$, and since $\mathrm{P}^{* *} \simeq \mathrm{Q}^{* *}$, these are isomorphic reductions of structure group of the principal bundle $\mathbb{E}_{U}$. Therefore they are in the same orbit of Aut $\left(\mathbb{E}_{U}\right)$. By the definition of the action in Remark 6.9, this implies that $P$ and $Q$ lie in the same orbit of Aut $(\mathbb{E})$.

Now since $\mathbb{E}$ is a polystable vector bundle on $X$ (we confuse the principal bundle with the vector bundle associated to it) by an argument similar to the one in Lemma 5.16, it follows that for a general curve $C \in X$, Aut $(\mathbb{E}) \simeq$ Aut $\left(\left.\mathbb{E}\right|_{C}\right)$. Also by the Mehta-Ramanathan restriction theorem, $\left.\mathbb{E}\right|_{C}$ is also a polystable vector bundle on $C$ and hence its automorphism group is a product of $G L(n)$ 's. Hence, Aut $(\mathbb{E})$ is connected. This implies that the orbit of Aut $(\mathbb{E})$ is connected and hence both $\mathrm{P}$ and $\mathrm{Q}$ lie in the same connected component of the fibre of $h$.

q.e.d.

Corollary 6.11. Let $\mathrm{P}$ and $\mathrm{Q}$ be two $\mu$-semistable $H$-quasibundles in $Q_{H}(\mathbb{E}, l)$ lying in the same fibre of $f_{\rho}$. If $\mathrm{P}_{0}^{* *} \simeq \mathrm{Q}_{0}^{* *}$ then $\mathrm{P}$ and $\mathrm{Q}$ lie in the same connected component of the fibre of the map $h: Q_{H}(\mathbb{E}, l) \longrightarrow$ $S^{l}(X)$. 
Proof. Observe that for any quasibundle $\mathrm{P}$, an associated graded quasibundle is connected to $\mathrm{P}$ by a path (cf. Def. 5.17). Hence the corollary follows from the Prop. 6.10.

Proposition 6.12. Let $\mathrm{P}$ and $\mathrm{Q}$ be two $\mu$-polystable $H$-quasibundles in $Q_{H}(\mathbb{E}, l)$ lying in the same fibre of $h$. If, further, $\mathrm{P}^{* *} \simeq \mathrm{Q}^{* *}$, then $\mathrm{P}$ and $\mathrm{Q}$ lie in the same connected component of the fibre of the map $h: Q_{H}(\mathbb{E}, l) \longrightarrow S^{l}(X)$ (or equivalently of the map $h_{0}: Q_{H}(\mathbb{E}, l) \longrightarrow$ $\left.S^{l_{0}}(X)\right)$.

Proof. By the result of of Baranovsky ([5]) and Ellingsrud and Lehn ([15]) (and in rank 2, Li ([26, Prop. 6.5])), one knows that the fibre of the map $q$, which we denote by $T$, is connected, and in fact irreducible. In particular, every torsion-free sheaf $\mathcal{F}$ in the fibre of $q$ is a sheaf such that $\mathcal{F}^{* *} \simeq \mathbb{E}$ and since $\mathbb{E}$ is polystable, it follows that so is $\mathcal{F}$. In other words, every closed point of the fibre $T$ is represented by a polystable torsion-free sheaf.

Let us fix the polystable quasibundle $\mathrm{P}$ in the fibre of $h$ and let $\mathrm{Q}$ be another polystable quasibundle such that $\mathrm{Q}^{* *} \simeq \mathrm{P}^{* *}$. The polystable torsion-free sheaves associated to $\mathrm{P}$ and $\mathrm{Q}$, namely $\mathcal{E}$ and $\mathcal{F}$ are in $T$. If $\mathcal{E}$ and $\mathcal{F}$ coincide then the result follows from Prop. 6.10. Let $f_{\rho}(\mathrm{P})=t_{0}$ and $f_{\rho}(\mathrm{Q})=t_{1}$. Since $T$ is irreducible, without loss of generality we may take $T$ to be an irreducible curve joining $t_{0}$ and $t_{1}$. Let $\bar{T}$ be its normalization. Hence, $\bar{T}$ is a smooth irreducible curve.

We claim that $\mathrm{Q}$ lies in the same connected component of the fibre of $h$ which contains $\mathrm{P}$.

Consider the family of torsion-free sheaves $\mathcal{E}_{T}$ (on $X \times T$ ) such that $\mathcal{E}_{t_{0}} \simeq \mathcal{E}$ and $\mathcal{E}_{t_{1}} \simeq \mathcal{F}$, which exists by the definition of the Quot scheme of torsion quotients. We shall work with the pull-back of $\mathcal{E}_{T}$ to $X \times \bar{T}$ and denote it by $\mathcal{E}_{\bar{T}}$. Thus we may take $t_{0}, t_{1} \in \bar{T}$.

Since all the sheaves $\mathcal{E}_{t}$ lie over the same fibre $T$ it follows that they are all polystable sheaves and have the same double dual $\mathbb{E}$. Let $\mathcal{U}$ be the maximal open subset where $\mathcal{E}_{\bar{T}}$ is locally free. Then we have an isomorphism of families on $\mathcal{U}$ :

$$
\left.\left.\mathcal{E}_{\bar{T}}\right|_{\mathcal{U}} \simeq \mathbb{E}_{\bar{T}}\right|_{\mathcal{U}}
$$

where $\mathbb{E}_{\bar{T}}=\mathbb{E} \times \bar{T}$.

Since the quasibundle $\mathrm{P}$ is a "reduction of structure group" of $\mathcal{E}_{t_{0}}$, it implies that there is a section $\sigma: X \longrightarrow\left(S_{\mathcal{E}_{t_{0}}}\right) / / H$.

Restricting this section to $\mathcal{U}_{t_{0}}=\mathcal{U} \cap\left(X \times t_{0}\right)$ we get a section $\sigma_{\mathcal{U}_{t_{0}}}$ : $\mathcal{U}_{t_{0}} \longrightarrow\left(S_{\mathcal{E}_{t_{0}}}\right) / / H$. Note that $\mathcal{U}_{t_{0}}$ is also a big open subset of $X$ (cf. $[\mathbf{3 7}, 2.6$,page 1187]).

Observe that the double dual principal bundle $\mathrm{P}^{* *}$ of $\mathrm{P}$ is also obtained by a reduction of structure group of the principal bundle associated to $\mathbb{E}$. 
Let us denote this reduction by $\tau: X \longrightarrow\left(S_{\mathbb{E}}\right) / H$. On the big open set $\mathcal{U}_{t_{0}}$ this reduction $\tau_{\mathcal{U}_{t_{0}}}$ and the reduction $\sigma_{\mathcal{U}_{t_{0}}}$ giving $\mathrm{P}$ are therefore mapped to each other by an automorphism $\phi \in \operatorname{Aut}\left(\mathbb{E}{\mid \mathcal{U}_{t_{0}}}\right)=\operatorname{Aut}(\mathbb{E})$.

Since $\mathbb{E}_{\bar{T}}=\mathbb{E} \times \bar{T}$, the reduction section $\tau$ trivially extends to a section $\tau_{\bar{T}}: X \times \bar{T} \longrightarrow\left(S_{\mathbb{E}_{\bar{T}}}\right) / H$. Restricting $\tau_{\bar{T}}$ to $\mathcal{U}$ we get a section

$$
\tau_{\mathcal{U}}: \mathcal{U} \longrightarrow\left(S_{\mathbb{E}_{\bar{T}} \mid \mathcal{U}}\right) / / H \simeq\left(S_{\mathcal{E}_{\bar{T}}}\right) / / H .
$$

Since $\mathcal{U}$ is big, and since $X \times \bar{T}$ is smooth (and hence normal), this section extends to give a new section $\bar{\tau}: X \times \bar{T} \longrightarrow\left(S_{\left.\mathcal{E}_{\bar{T}}\right|_{X \times \bar{T}}}\right) / / H$, i.e., a "reduction datum" for the family $\mathcal{E}_{\bar{T}}$ (see Remark 2.5).

This gives a family $\overline{\mathrm{P}}_{\bar{T}}$ of quasibundles on $X \times \bar{T}$ such that $\overline{\mathrm{P}}_{t_{0}}$ and $\mathrm{P}$ are in the same orbit of Aut $(\mathbb{E})$ (by the element $\phi$ ). Thus $\overline{\mathrm{P}}_{t_{0}}$ and $\mathrm{P}$ lie in the same connected component of the fibre of $h$.

By the universal property of $Q_{H}(\mathbb{E}, l)$, we get a morphism induced by this family of quasibundles, namely

$$
\psi: \bar{T} \longrightarrow Q_{H}(\mathbb{E}, l)
$$

( $\psi$ depends on the choice of the reduction $\tau$ ).

Further, by the definition of the reduction datum on $\mathcal{U}$, all the quasibundles in the family $\overline{\mathrm{P}}_{\bar{T}}$ (which are polystable) have isomorphic double duals $\mathrm{P}^{* *}$.

In particular, they lie in the same connected component of the fibre of $h$. Moreover, if $\mathrm{Q}$ corresponds to the point $t_{1} \in \bar{T}$ then the quasibundles $\overline{\mathrm{P}}_{t_{1}}$ and $\mathrm{Q}$ lie in the same fibre $f_{\rho}^{-1}\left(\mathcal{E}_{t_{1}}\right)$. By Prop. 6.10 they lie in the same orbit of Aut $(\mathbb{E})$ since $Q^{* *} \simeq P^{* *}$. This implies that the quasibundles $\mathrm{P}, \overline{\mathrm{P}}_{t_{0}}, \overline{\mathrm{P}}_{t_{1}}$ and $\mathrm{Q}$ lie on the same connected component of the fibre of $h$. This proves the claim.

q.e.d.

6.2.1. Characteristic classes of principal $H$-bundles. Let $P$ be a principal $H$-bundle with $H$ a simple algebraic group. Then by the tables in [8] for the "invariant degrees", i.e., the degrees of a generating set for the invariant polynomials under the adjoint action (or equivalently by the Chevalley restriction theorem, polynomials on the Cartan subalgebra invariant under the Weyl group action), we see that for $H$ simple, there is a unique generator of degree 2. Since we are over an algebraic surface, it follows that this invariant polynomial $I_{2}$ will be the only one which gives us a characteristic class in $H^{4}(X, \mathbb{Z})$. We shall denote this class by $c(P)$ (cf. $[6])$.

Example 6.13. When $H$ is classical, then $c(P)=c_{2}(P(V))$ where $V$ is the defining representation.

We can now state the following key result:

Proposition 6.14. Let $\mathrm{P}$ and $\mathrm{Q}$ be two $\mu$-semistable $H$-quasibundles. Let $\mathrm{P}_{0}^{* *}$ be the canonical polystable principal bundle obtained from a 
choice of associated graded quasibundle of $\mathrm{P}$. Let $c\left(\mathrm{P}_{0}^{* *}\right)=c\left(\mathrm{Q}_{0}^{* *}\right)$ for the degree 2 characteristic class $c$ of the principal bundles $\mathrm{P}_{0}^{* *}$ and $\mathrm{Q}_{0}^{* *}$. Then $\mathrm{P}$ and $\mathrm{Q}$ define the same point of $M_{H}(\rho)$ if and only if we have an identification of pairs $\left(\mathrm{P}_{0}^{* *}, Z(\mathrm{P})\right) \simeq\left(\mathrm{Q}_{0}^{* *}, Z(\mathrm{Q})\right)$ where $Z(\mathrm{P})$ is a cycle class in the symmetric power $S^{l_{0}}(X)$ given by

$$
Z(\mathrm{P}):=\sum_{x \in X} \frac{\text { length }\left(\mathcal{E}_{0}^{* *} / \mathcal{E}_{0}\right)_{x}}{m_{\rho}} \cdot x
$$

with $l_{0}$ given by:

$$
l_{0}=\frac{c_{2}\left(\mathcal{E}_{0}\right)-c_{2}\left(\mathcal{E}_{0}^{* *}\right)}{m_{\rho}}
$$

and where $\mathrm{P}($ resp $\mathrm{Q})$ is obtained from the torsion-free sheaf $\mathcal{E}$ (resp $\mathcal{F}$ ) by reduction of structure group via $\rho$ and $\mathcal{E}_{0}\left(\right.$ resp $\left.\mathcal{F}_{0}\right)$ is an associated graded sheaf of the semistable sheaf $\mathcal{E}($ resp $\mathcal{F})$.

Proof. Let us assume that the quasibundles $\mathrm{P}$ and $\mathrm{Q}$ define the same point in the moduli space $M_{H}(\rho)$.

We first check that $\mathrm{P}_{0}^{* *} \simeq \mathrm{Q}_{0}^{* *}$ : suppose that this does not hold. As we noted in Lemma 5.16, it is implied that for a large degree curve $C$, the restrictions $\left.\mathrm{P}_{0}^{* *}\right|_{C} \neq\left.\mathrm{Q}_{0}^{* *}\right|_{C}$. By Lemma 5.6, we can separate the points corresponding to $\mathrm{P}$ and $\mathrm{Q}$ in $R_{H}(\rho)$ by invariant sections of $\mathcal{L}$, which contradicts the assumption that they define the same point in $M_{H}(\rho)$.

Again, the equality $Z(\mathrm{P})=Z(\mathrm{Q})$ follows immediately since the torsion-free sheaves $\mathcal{E}$ and $\mathcal{F}$ (from which the quasibundles $\mathrm{P}$ and $\mathrm{Q}$ are defined) give the same point in the moduli space $M_{G}$ by $([\mathbf{2 1}, \mathrm{Th}$. 8.2.11]), and the cycle classes $m_{\rho} \cdot Z(\mathrm{P})=Z(\mathcal{E})$.

For the converse, suppose that we have an identification:

$$
\left(\mathrm{P}_{0}^{* *}, Z(\mathrm{P})\right) \simeq\left(\mathrm{Q}_{0}^{* *}, Z(\mathrm{Q})\right) .
$$

By Cor. 6.11, it is enough to check for polystable quasibundles in the fibre of $h$; by Prop. 6.12 it follows that $\mathrm{P}$ and $\mathrm{Q}$ lie in the same connected component of the fibre of $h: Q_{H}(\mathbb{E}, l) \longrightarrow S^{l}(X)$.

By the definition of the determinant line bundle on $R_{H}(\rho)$, it is obtained by pulling back the corresponding line bundle from the total family $R_{G}$. By the theorem of Baranovsky $([5])$ and Ellingsrud and Lehn ([15]) one knows that the fibres of the morphism $q: Q(\mathbb{E}, l) \longrightarrow S^{l}(X)$ are connected and also that the line bundle $\mathcal{L}$ restricted to this fibre is trivial (cf. [21, 8.2.1] and Li ([26, Lemma 3.4,3.5])).

By the commutative diagram seen earlier, we have $q \circ f_{\rho}=h$. Therefore $\mathcal{L}$ is trivial on the fibres of $h$ as well. In particular, the connected components of the fibre of $h$ get mapped to the same point in $M_{H}(\rho)$. This proves that the points defined by $\mathrm{P}$ and $\mathrm{Q}$ coincide in $M_{H}(\rho)$. 
Proposition 6.15. There is a canonical morphism $j: M_{H}(\mathrm{c})^{s} \longrightarrow$ $M_{H}(\rho)$ which is an embedding of the moduli space of isomorphism classes $\mu$-stable principal $H$-bundles in $M_{H}(\rho)$.

Proof. The existence of the morphism $j$ follows by the weak coarse moduli property of the moduli space $M_{H}(\rho)$. The injectivity of $j$ follows from Lemma 5.16.

Now choose a general curve $C \in|a \Theta|$ which gives Bogomolov's effective restriction theorem; it is easily seen that the differential of the restriction map $\left.r\right|_{C}$ (by usual arguments involving Enriques-Severi lemma) is injective. This immediately implies that the map $j$ is an embedding. q.e.d.

Let $\mathfrak{M}_{H}$ be defined by the disjoint union:

$$
\mathfrak{M}_{H}=\coprod_{l \geq 0} M_{H}^{\mu-p o l y}(\mathrm{c}-l) \times S^{l}(X)
$$

where $M_{H}^{\mu-p o l y}(\mathrm{c}-l)$ is the moduli space of $\mu$-semistable principal $H$-bundles with characteristic class $\mathrm{c}-l$ (represented as classes of polystable bundles); in particular, the big stratum is $M_{H}^{\mu-p o l y}(\mathrm{c})=M_{H}^{0}$.

In conclusion we have the following:

Theorem 6.16. There is a set-theoretic inclusion

$$
\mathfrak{M}_{H} \subset M_{H}(\rho)(\mathbb{C}) .
$$

Furthermore, the underlying set of points $\overline{M_{H}^{0}}(\mathbb{C})$, of the closure of the moduli space of equivalence classes of $\mu$-semistable principal $H$-bundles, is a subset of $\mathfrak{M}_{H}$. In fact, $\overline{M_{H}^{0}}(\mathbb{C})$ is independent of the representation $\rho$ up to homeomorphism.

Proof. To complete the proof of the theorem we need only show that if $\left(\mathrm{P}^{* *}, Z\right)$ is a point on the right hand side of $(*)$ above, then there exists a point in $M_{H}(\rho)$ which corresponds to it.

Since $\mathrm{P}^{* *}$ is a polystable principal $H$-bundle, using $\rho$ if we extend the structure group to $G=G L(V)$, we get a $\mu$-polystable locally free sheaf $\mathbb{E} \in M_{G}$ together with a reduction $\sigma: X \longrightarrow \mathbb{E}(G / H)$ (as before we confuse the locally free sheaf $\mathbb{E}$ with the associated principal $G L(V)$ bundle $\mathbb{E}$ ).

By the results of $\mathrm{Li}$, it is known that the map $j: S^{l}(X) \longrightarrow M_{G}$, induced by the morphism from $Q(\mathbb{E}, l) \longrightarrow M_{G}$, is an embedding. Therefore the element $m_{\rho} \cdot Z \in S^{l}(X)$ gives a polystable torsion-free sheaf $\mathcal{E}$ such that $\mathcal{E}^{* *} \simeq \mathbb{E}$ and such that $m_{\rho} \cdot Z=Z(\mathcal{E})$.

We therefore have a reduction of structure group of $\left.\mathcal{E}\right|_{U(\mathcal{E})}$ coming from the restriction $\left.\sigma\right|_{U(\mathcal{E})}$ of the reduction of structure group of $\mathbb{E}$ which gives the principal bundle $\mathrm{P}^{* *}$. Viewing this $\left.\sigma\right|_{U(\mathcal{E})}$ as a morphism $U(\mathcal{E}) \longrightarrow S_{\mathcal{E}} / / H$, we get a canonical extension $\sigma_{1}: X \longrightarrow S_{\mathcal{E}} / / H$. This 
gives rise to a polystable $H$-quasibundle $\mathrm{P} \in M_{H}(\rho)$ which corresponds to the point $\left(\mathrm{P}^{* *}, Z\right)$. That this map which sends $\left(\mathrm{P}^{* *}, Z\right) \longrightarrow \mathrm{P}$ is injective follows from the first part of the proof of Prop. 6.14.

The last part of the theorem which realizes the closure $\overline{M_{H}^{0}}(\mathbb{C})$ in $\mathfrak{M}_{H}$ follows from the consequences of Theorem 6.7 and Definition 6.8. The uniqueness up to homeomorphism is precisely the content of Prop. 6.17 below.

q.e.d.

Proposition 6.17. The closure $\overline{M_{H}^{0}}(\mathbb{C})$ is unique up to homeomorphism. In fact, the isomorphic copies of $M_{H}^{0}$ define a correspondence between the closures which gives the homeomorphism. Furthermore, the normalizations of the closures are isomorphic as reduced projective schemes.

Proof. Since most of the points needed in the proof have already been discussed above, we content ourselves by giving the main steps in the proof of this proposition.

Let $\rho_{i}: H \hookrightarrow S L\left(V_{i}\right)$ for $i=1,2$ be two faithful representations of $H$ and suppose that $P_{t}(i)$ is a family of $\mu$-semistable principal $H$ bundles with isomorphic generic fibres and with two limits as polystable $H$-quasibundles coming from the representations $\rho_{i}$. Let the limits be $P(i), i=1,2$.

Claim 1. The double dual polystable $H$-bundles $P(1)^{* *} \simeq P(2)^{* *}$. To see this, restrict the families $P_{t}(i)$ to a general high degree curve $C$ which avoids the singular loci of $P(i)$ for $i=1,2$. Then, since for such families over curves the (polystable) limit is uniquely defined, it follows that $\left.\left.P(1)^{* *}\right|_{C} \simeq P(2)^{* *}\right|_{C}$. By Lemma 5.16 the claim follows.

Claim 2. The cycles $Z(P(i))$ also coincide. This follows from two observations, namely that for the limits the open subsets where the $P(i)$ are genuine principal bundles coincide and hence the sets $\operatorname{Sing}(P(i))_{\text {red }}$ coincide. The multiplicities at all the singular points also coincide by the discussion before Definition 6.8.

These two claims imply the set-theoretic identification of the closures. Moreover, the above discussion gives an identification of closures of any curve in $M_{H}^{0}$. This implies that the projections to $M_{H}\left(\rho_{i}\right)$ from the graph $\Gamma \subset M_{H}\left(\rho_{1}\right) \times M_{H}\left(\rho_{2}\right)$, which is closed in the product, are proper and bijective and hence actually give homeomorphisms. The comment on normalizations now follows by Zariski's main theorem.

Corollary 6.18. The closure of the subset $M_{H}(\mathrm{c})^{s} \subset M_{H}^{0}$ gives the Donaldson-Uhlenbeck compactification of the moduli space of principal $H$-bundles with irreducible ASD connections.

6.2.2. Relationship with Gómez-Sols and Schmitt's moduli space. We work in the set-up of Schmitt's recent paper (cf. [37] and 
[38]). Fix a faithful representation $\rho: H \hookrightarrow S L(V)$. Define the moduli functors

$$
\underline{M}(\rho)_{P}^{s S}: \underline{S c h} \mathbb{C} \longrightarrow \underline{\text { Set }}
$$

which sends $S$ to "equivalence classes of families of Gieseker-Maruyama semistable honest singular principal $H$-bundles (or $H$-quasibundles) with Hilbert polynomial $\mathrm{P}$ on $X$ parametrised by $S$ ". Then the main theorem of $[\mathbf{3 8}]$ is that there is a projective scheme $\mathcal{M}(\rho)_{P}^{s s}$ which coarsely represents the functor $\underline{M}(\rho)_{P}^{s s}$. The notions of Gieseker-Maruyama semistability of quasibundles is defined in [19] and [38] and it is also shown that if $\mathrm{P}=(\mathcal{E}, \tau)$ is an $H$-quasibundle then the following equivalence holds ([38, Section 5.1]): $\mathrm{P}$ is $\mu$ - stable $\Longrightarrow$ $\mathrm{P}$ is Gieseker-Maruyama-stable $\Longrightarrow \mathrm{P}$ is Gieseker-Maruyamasemistable $\Longrightarrow \mathrm{P}$ is $\mu$-semistable. These implications, together with the coarse moduli property of the functor $\underline{M}(\rho)_{P}^{s s}$ implies that there is a morphism:

$$
\mathcal{M}(\rho)_{P}^{s s} \longrightarrow M_{H}(\rho)
$$

At the risk of repetition, we now summarize the above results in the following:

Theorem 6.19. Let $H$ be a semisimple algebraic group.

(a) There exists a projective scheme $M_{H}(\rho)$ which parametrizes equivalence classes of $\mu$-semistable $H$-quasibundles with fixed characteristic classes, on the smooth projective surface $X$.

(b) This has an open subscheme of equivalence classes $\mu$-semistable principal $H$-bundles $M_{H}^{0}$.

(c) If $M_{H}^{s}$ is the subscheme of $M_{H}(\rho)$ consisting of the stable principal $H$-bundles, then its closure $\overline{M^{s}}$ in $M_{H}(\rho)$ corresponds to the Donaldson-Uhlenbeck compactification of the moduli space of $\mu$-stable vector bundles on $X$ (or equivalently of anti self-dual connections).

(d) When $H$ is a simple algebraic group the points of the moduli space $\overline{M_{H}^{0}}$ are given by the pairs $\left(\mathrm{P}_{0}^{* *}, Z(\mathrm{P})\right)$ where, to each semistable $H$-quasi bundle $\mathrm{P}$, we associate $\mathrm{P}_{0}^{* *}$ the canonical polystable principal $H$-bundle obtained from the quasibundle $\mathrm{P}$ and the cycle class $Z(\mathrm{P})$ which lies in $S^{l_{0}}(X), l_{0}$ being given by:

$$
\frac{c_{2}\left(\mathcal{E}_{0}\right)-c_{2}\left(\mathcal{E}_{0}^{* *}\right)}{m_{\rho}}
$$

Note that $\mathrm{P}$ is obtained from the torsion-free sheaf $\mathcal{E}$ by reduction of structure group via $\rho$ and $\mathcal{E}_{0}=g r^{\mu}(\mathcal{E})$ and $m_{\rho}$ is the Dynkin index associated to $\rho$.

(e) When $H$ is a simple algebraic group the underlying set of points of the moduli space $\overline{M_{H}^{0}}$, up to homeomorphism, is independent of the representation $\rho$. 
(f) $B y[\mathbf{3 8}$, Main theorem], we have a morphism

$$
\mathcal{M}(\rho)_{P}^{s s} \longrightarrow M_{H}(\rho) \text {. }
$$

(g) In a certain sense, $\overline{M_{H}^{0}}$ is the minimal compactification of $M_{H}^{0}$.

\section{Non-emptiness of the moduli space}

In this section $H$ is an arbitrary semisimple algebraic group. The aim of this section is to prove that the moduli space $M_{H}(\mathrm{c})^{\mathrm{s}}$ of $\mu$-stable principal $H$-bundles on a smooth projective surface $X$ for large characteristic classes c is non-empty. When $H=S L(2)$ this is a theorem due to Taubes [43] and later due to Gieseker [17]. We quote their result:

Theorem 7.1 (Taubes, Gieseker). For any choice of polarization $\Theta$, for all $c \geq 2 p_{g}(X)+2$, the moduli space $M_{S L(2)}(c)^{s}$ of stable rank 2 bundles with trivial determinant and $c_{2}=c$ is non-empty.

Remark 7.2. We remark that this result of Taubes and Gieseker gives the existence of $\mu$-stable rank two vector bundles with a purely topological bound on $c_{2}$ that does not depend on the polarization. Such an existence result is of importance in the computation of Donaldson invariants where the independence of the choice of the metrics plays a key role. What we prove below (Theorem 7.10) for a general semisimple group $H$ is also with bounds which are independent of the polarization. We refer the reader to $[\mathbf{2 1}$, Chapter 5] for other proofs of existence of $\mu-$ stable bundles but with bounds dependent on the choice of polarization. 7.1. Stable bundles and the principal three dimensional subgroup. We begin by recalling a few facts about semisimple Lie algebras. Let $\mathfrak{H}=\operatorname{Lie}(H)$. Then one knows that there exist the so-called principal $\mathfrak{s l}(2)$ 's inside $\mathfrak{H}$ which are distinguished by the property that they do not lie in any parabolic subalgebra of $\mathfrak{H}$. This gives a representation $\phi: S L(2) \rightarrow H$ which is irreducible, in the sense that $\operatorname{Im}(\phi)$ is not contained in any proper parabolic subgroup of $H$ (cf. [8] Chapter 8, Exercise $5 \S 11$, p. 246).

We fix one such homomorphism of a principal $S L(2) \rightarrow H$.

Let $C$ be a smooth projective curve of genus $g \geq 2$. Let $V$ be a stable vector bundle of rank 2 . Then by the Narasimhan-Seshadri theorem one knows that there exists an irreducible representation $\rho: \pi_{1}(C) \longrightarrow$ $S U(2)$ such that $V \simeq V_{\rho}$.

Definition 7.3. We define the monodromy subgroup $\mathcal{M}(V)$ of $V \simeq$ $V_{\rho}$ to be the Zariski closure of $\operatorname{Im}(\rho)$ in $S L(2)$. It can be viewed as the minimal subgroup to which the structure group of $V$ can be reduced.

Remark 7.4. Recall that one knows that the reductivity of $\mathcal{M}(V)$ is equivalent to the polystability of $V$. Since one knows the set of all finite subgroups of $S L(2)$ (this is classical, for example cf. [40]), the only possibilities for $\mathcal{M}(V)$ are the following: 
(a) Finite cyclic groups $C_{n}$ and the dihedral groups $D_{n}$.

(b) The alternating groups $A_{4}$ and $A_{5}$ and the permutation group $S_{5}$.

(c) The whole of $S L(2)$ or its maximal torus.

Of these, since $V$ is stable, we can omit the maximal torus and the cyclic groups $C_{n}$. So $\mathcal{M}(V)$ can either be the alternating groups, $S_{5}$, or the dihedral groups apart from the whole of $S L(2)$.

We wish to estimate the set $Z_{C}$ of representations of $\pi_{1}(C)$ in $S L(2)$ which lie entirely in these families of finite groups up to conjugacy by the diagonal action of $S L(2)$.

It is not hard to see that since $\pi_{1}(C)$ is given by $2 g$ generators with a single relation, this set $Z$ is at most countable. This implies the following lemma.

Lemma 7.5. The locus of points in the moduli space of stable vector bundles $M_{C, S L(2)}$ of rank 2 and trivial determinant on the curve $C$ whose monodromy is among the set of finite subgroups listed above has countable cardinality.

Using this we have the following:

Proposition 7.6. There exists a rank 2 stable bundle $E$ with $c_{2}(E) \gg$ 0 and trivial determinant on the surface $X$ such that the restriction $\left.E\right|_{C}$ to general curve $C \subset X$ of high degree has monodromy subgroup to be the whole of $S L(2)$ itself.

Proof. For $c=c_{2}(E) \gg 0$ (by Theorem 7.1), it is known that the moduli space $M_{X}\left(2, \mathcal{O}, c_{2}\right)^{s}=M_{S L(2)}(c)^{s}$ is non-empty and has dimension $d(c)=4 c-3 \chi\left(\mathcal{O}_{X}\right)$. Since $c_{2}(E) \gg 0$, it follows that we can make $d \geq 1$.

Now we consider the restriction map $r_{C}: M_{S L(2)}(c)^{s} \longrightarrow M_{C, S L(2)}^{s}$. If the curve $C$ is chosen sufficiently general and high degree $k$ then by the Mehta-Ramanathan theorem and [16, Prop. 2.2], it can be seen that for $k \gg 0$, the map $r_{C}$ is in fact an immersion. This implies that $\operatorname{Im}\left(r_{C}\right) \subset M_{C, S L(2)}^{s}$ is not completely contained in the subset $Z_{C}$ of stable bundles with finite monodromy groups. Therefore there exists at least one stable bundle with the whole of $S L(2)$ as its monodromy subgroup. This proves the proposition.

Before we proceed to our next lemma we recall the following formulation of irreducible sets ([34, p. 129]):

Definition 7.7. Let $K$ be a maximal compact subgroup of $H$. A subset $A \subset K$ is said to be irreducible if

$$
\{Y \in \mathfrak{H} \mid a d x(Y)=Y, \forall x \in A\}=\text { center of } \mathfrak{H} \text {. }
$$

A (homomorphism) representation $\delta: \Gamma \longrightarrow K$ is said to be irreducible if the image $\delta(\Gamma)$ is irreducible. 
Note that in our case, since $H$ is semisimple the center of $\mathfrak{H}$ is trivial; we have the following equivalences and we quote:

Proposition 7.8 ([34, Prop. 2.1]). Let $K$ be a maximal compact subgroup of $H$ and let $A \subset K$ be a subgroup. Then the following are equivalent:

(a) $A$ is irreducible.

(b) A leaves no parabolic subalgebra of $\mathfrak{H}$ invariant.

(c) For any parabolic subgroup $Q \subset H, A$ acts without fixed points on $H / Q$.

Lemma 7.9. If $V$ is a stable bundle on a curve $C$ such that $\mathcal{M}(V)=$ $S L(2)$ then $V(\phi)$ is a stable $H$-bundle on $C$ by an extension of structure group via the principal homomorphism $\phi$.

Proof. Let $\rho: \pi_{C} \longrightarrow S U(2)$ be an irreducible unitary representation and let $V \simeq V_{\rho}$ be a stable bundle on $C$ such that $\mathcal{M}(V)=S L(2)$.

This implies that $\operatorname{Im}(\rho)$ is Zariski dense in $S L(2)$. Since $\operatorname{Im}(\phi)$ is not completely contained in any parabolic subgroup of $H$ by Prop. 7.8 we have the following:

$$
\{Y \in \mathfrak{H} \mid \text { ad } x(Y)=Y, \forall x \in \mathfrak{s l}(2)\}=\text { centre of } \mathfrak{H}=\text { trivial. }
$$

In other words, we can say that $\operatorname{Im}(\phi)$ is an irreducible subset in $H$ (or equivalently, by the "unitarian trick", $\operatorname{Im}\left(\left.\phi\right|_{\mathfrak{s u}(2)}\right)$ is an irreducible subset of $K$, where, as above, $K \subset H$ is a maximal compact subgroup).

By [34, Prop. 2.2], to show that $V(\phi)$ is stable as an $H$-bundle, we need only show that the representation

$$
\eta=(\phi \circ \rho): \pi_{1}(C) \longrightarrow K
$$

is irreducible.

If $Y \in \mathfrak{H}$ is such that $a d x(Y)=Y, \forall x \in \operatorname{Lie}(\operatorname{Im}(\eta))$, then by the density of $\operatorname{Im}(\eta)$ in $\operatorname{Im}(\phi)=\mathfrak{s l}(2)$ and hence by continuity, we see that for such an element $Y$, one has $a d x(Y)=Y, \forall x \in \mathfrak{s l}(2)$ and hence $Y \in$ centre of $\mathfrak{H}$. This shows that $\operatorname{Im}(\eta)$ is an irreducible set in $K \subset H$ and we are done.

q.e.d.

We can now conclude the following:

Theorem 7.10. The moduli space $M_{H}(c)^{s}$ of $\mu$-stable principal $H$ bundles on a smooth projective surface $X$ is non-empty for $c>\delta$, where $\delta$ depends only on $p_{g}(X)$ and not on the polarization $\Theta$ on $X$.

Proof. We claim that if $\phi: S L(2) \longrightarrow H$ is as above a principal $S L(2)$ in $H$ then any $E$ as in Prop. 7.6 has the property that $E(\phi)$ is a stable principal $H$-bundle.

By the converse to the Mehta-Ramanathan theorem (Lemma 4.5), we see that it is enough to prove that $\left.E(\phi)\right|_{C}$ is stable. Since $\mathcal{M}\left(\left.E\right|_{C}\right)=$ $S L(2)$, this is immediate by Lemma 7.9. The largeness of the characteristic classes of the associated $H$-bundle is determined by the largeness 
of the $c_{2}$ of the rank 2 bundle $E$. This can determined by the general methods of Borel and Hirzebruch (see the recent preprint Beauville [6] for this).

\subsection{Concluding remarks.}

q.e.d.

(a) The questions addressed in this paper can be posed in positive characteristic as well, but as in the case of curves a subtler analysis of representation theoretic bounds such as the ones considered in [4] will have to be carried out. We hope to do this in future. We remark that after this paper was circulated (in 2004) and posted in 2005 , the preprint $([\mathbf{2 0}])$ has been posted which proposes to solve the Maruyama-Gieseker moduli construction over fields of arbitrary characteristic over a smooth projective variety.

(b) The basic questions regarding irreducibility, reducedness, generic smoothness and normality are yet to be answered for these moduli spaces.

(c) We refer the reader to the preprint of Drinfeld ([13, p. 28]) where the problem of existence of the Uhlenbeck spaces on arbitrary algebraic surfaces is discussed. Drinfeld proposes an alternative object which he calls a $H$-gundle that is expected to play the role of our $H$-quasibundles.

(d) One may consider, more generally, the algebraic stack $B u n_{X}\left(S L_{n}\right)$ of torsion-free sheaves of rank $n$ and trivial determinant over a smooth projective algebraic variety $X$. By the definition of an $H$-quasibundle given in Def. 2.2, since giving $\mathrm{P}$ is equivalent to giving a pair $(\mathcal{E}, \sigma)$, by the general theory of Hilbert schemes we have an algebraic stack of $H$-quasibundles. Our Theorem 1.1 in fact proves that the substack of semistable $H$-quasibundles is proper. The construction of the compactified Donaldson-Uhlenbeck moduli space (with its reduced structure) can also be defined for arbitrary $X$, but the key issue required for any application, namely the description of boundary points, is very hard. There is a recent paper by G. Tian ([44]) solving this in general in the differential geometric setting for a compact Riemannian manifold. The corresponding algebro-geometric problem is open to the best of our knowledge.

\section{References}

[1] M.F. Atiyah \& R. Bott, The Yang-Mills equations over Riemann Surfaces, Phil. Tr. R. Soc. Lond. A 308 (1982) 523-615, MR 0702806, Zbl 0509.14014.

[2] M. Atiyah, N. Hitchin, \& I.M. Singer, Self-duality in four dimensional Riemannian geometry, Proc. Royal Soc. Lond. A 362 (1978) 425-461, MR 0506229, Zbl 0389.53011.

[3] V. Balaji \& C.S. Seshadri, Semistable Principal Bundles-I (in characteristic zero), Journal of Algebra 258 (2002) 321-347, MR 1958909.

[4] V. Balaji \& A.J. Parameswaran, Semistable principal bundles-II (in positive characteristics), Transformation Groups 18(1) (2003) 3-36, MR 1959761. 
[5] V. Baranovsky, Moduli of sheaves on surfaces and action of the oscillator algebra, J. Differential Geom. 55(2) (2000) 193-2, MR 1847311, Zbl 1033.14028.

[6] A. Beauville, Chern classes for representations of reductive groups, math.AG/ 0104031.

[7] I. Biswas and T.L. Gómez, Restriction theorem for principal bundles, Math. Annalen 327 (2003) 773-792, MR 2023316, Zbl 1071.14020.

[8] N. Bourbaki, Groupes et algèbres de Lie, Chap 7-8, Paris, Hermann, 1975, MR 0453824, Zbl 0329.17002.

[9] A. Braverman, M. Finkelberg, \& D. Gaitsgory, Uhlenbeck spaces via affine Lie algebras, The unity of mathematics, 17-135, Progr. Math., 244, Birkhuser Boston, Boston, MA, 2006, MR 2181803, math.AG/0301176.

[10] J.-L. Colliot-Thélène \& J.Sansuc, Fibrés quadratiques et composantes connexes réelles, Math.Annalen 244 (1979) 105-134, MR 0550842, Zbl 0418.14016.

[11] S.K. Donaldson, Polynomial invariants for smooth four manifolds, Topology 29 (1990) 257-315, MR 1066174, Zbl 0715.57007.

[12] S.K. Donaldson \& P.B. Kronheimer, The Geometry of Four-Manifolds, Oxford, 1990, MR 1079726, Zbl 0904.57001.

[13] V. Drinfeld, Infinite dimensional vector bundles in algebraic geometry, math. AG/0309155, 2004.

[14] E.B. Dynkin, Semisimple subalgebras of semisimple Lie algebras, Am Math Soc Transl. Ser II 6 (1957) 111-244, MR 0047629, Zbl 0077.03404.

[15] G. Ellingsrud \& M. Lehn, Irreducibility of the punctual quotient scheme of a surface, Ark. Mat. 37(2) (1999) 245-254, MR 1714770, Zbl 1029.14017.

[16] R. Friedman \& J. Morgan, Smooth four-manifolds and complex surfaces, Ergebnisse, Vol 27, Springer Verlag, 1994, MR 1288304, Zbl 0817.14017.

[17] D. Gieseker, A construction of stable bundles on an algebraic surface, J. Differential Geom. 27 (1988) 137-154, MR 0918460, Zbl 0648.14008.

[18] D. Gieseker \& J. Li, Moduli of high rank vector bundles over surfaces, J. AMS 9 (1996) 107-151, MR 1303031, Zbl 0864.14005.

[19] T. Gómez \& I. Sols, Moduli spaces of principal sheaves over projective varieties, Annals of Math. 161(2) (2005) 1037-1092, MR 2153406, Zbl 1079.14018.

[20] T.L. G'omez, A. Langer, A.H.W. Schmitt, \& I. Sols, Moduli Spaces for Principal Bundles in Arbitrary Characteristic, math.AG/0506511.

[21] D. Huybrechts \& M. Lehn, The Geometry of moduli spaces of sheaves, Aspects of Mathematics E31, Vieweg, Braunschweig/Wiesbaden, 1997, MR 1450870, Zbl 0872.14002.

[22] S. Kumar, M.S. Narasimhan, \& A. Ramanathan, Infinite Grassmanians and moduli spaces of $G$-bundles, Math. Ann. 300 (1994) 41-75, MR 1289830, Zbl 0803.14012.

[23] A. Langer, Semistable sheaves in positive characteristic, Annals of Math. 159(1) (2004) 251-276, MR 2051393, Zbl 1080.14014.

[24] S. Langton, Valuative criterion for families of vector bundles on algebraic varieties, Annals of Mathematics (2) 101 (1975) 88-110, MR 0364255, Zbl 0307.14007.

[25] J. Le Potier, Fibré déterminant et courbes de saut sur les surfaces algébriques, Complex projective Geometry, London Mathematical Society, Bergen, 1989, 213 240, MR 1201385, Zbl 0788.14045.

[26] J. Li, Algebraic geometric interpretation of Donaldson's polynomial invariants of algebraic surfaces, J. Differential Geom. 37 (1993) 416-466, MR 1205451, Zbl 0809.14006. 
[27] _ Compactification of moduli of vector bundles over algebraic surfaces, in a volume of papers dedicated to Prof C.G. Gu, MR 1635663, Zbl 1032.14500.

[28] M. Maruyama, The Theorem of Grauert-Mülich-Spindler, Math. Ann. 255 (1981) 317-333, MR 0615853, Zbl 0438.14015.

[29] V.B. Mehta \& A. Ramanathan, Restriction of stable sheaves and representations of the fundamental group, Invent.Math. 77 (1984) 163-172, MR 0751136, Zbl 0525.55012.

[30] J. Morgan, Comparison of the Donaldson polynomial invariants with their algebro geometric analogues, Topology 32 (1993) 449-488, MR 1231956, Zbl 0801.57014.

[31] M.S. Narasimhan \& C.S. Seshadri, Stable and unitary vector bundles on a compact Riemann surface, Annals of Mathematics (2) 82 (1965) 540-567, MR 0170350, Zbl 0171.04803.

[32] P. Newstead, Introduction to moduli problems, TIFR Lecture Notes in Mathematics, 1978, MR 0546290, Zbl 0411.14003.

[33] S. Ramanan \& A. Ramanathan, Some remarks on the instability flag, Tohoku Math. Journ. 36 (1984) 269-291, MR 0742599, Zbl 0567.14027.

[34] A. Ramanathan, Stable principal bundles on a compact Riemann surface, Math. Ann. 213 (1975) 129-152, MR 0369747, Zbl 0284.32019.

[35] - Stable principal bundles on a compact Riemann surface - Construction of moduli space, Thesis, Bombay University, 1976;

Proc. Ind. Acad. Sci 106 (1996) 301-328, MR 1420170, Zbl 0901.14007;

Proc. Ind. Acad. Sci 106 (1996) 421-449, MR 1425616, Zbl 0901.14008.

[36] A. Ramanathan \& S. Subramaniam, Einstein-Hermitian connections on principal bundles and stability, J. Reine Angew. Math. 390 (1988) 21-31, MR 0953674, Zbl 0648.53017.

[37] A. Schmitt, Singular Principal Bundles over higher dimensional manifolds and their moduli spaces, IMRN 23 (2002) 1183-1209, MR 1903952, Zbl 1034.14017.

[38] _ A closer look at semistability for singular principal bundles, IMRN 62 (2004) 3327-3366. MR 2097106.

[39] J.-P. Serre, Espaces fibrés algébriques, Anneaux de Chow et applications, Séminaire Chevalley, 1958, also Documents Mathématiques 1 (2001) 107-140, MR 1942136.

[40] - Moursund Lectures, University of Oregon, Mathematics Department, 1998.

[41] C.S. Seshadri, Geometric reductivity over arbitrary base, Advances in Mathematics 25 (1977), MR 0466154, Zbl 0371.14009.

[42] C. Simpson, Higgs bundles and Local systems, Pub. I.H.E.S. 75 (1992) 5-95. MR 1179076, Zbl 0814.32003.

[43] C.H. Taubes, Self-dual connections on 4-manifolds with indefinite intersection matrix, J. Differential Geom. 19 (1984) 517-560. MR 0755237, Zbl 0552.53011.

[44] G. Tian, Gauge theory and calibrated geometry, Annals of Math. 151 (2000) 193-268. MR 1745014, Zbl 0957.58013.

Chennai Mathematical Institute SIPCOT IT PARK, SiRuseri ChennaI-603103, InDIA

E-mail address: balaji@cmi.ac.in 